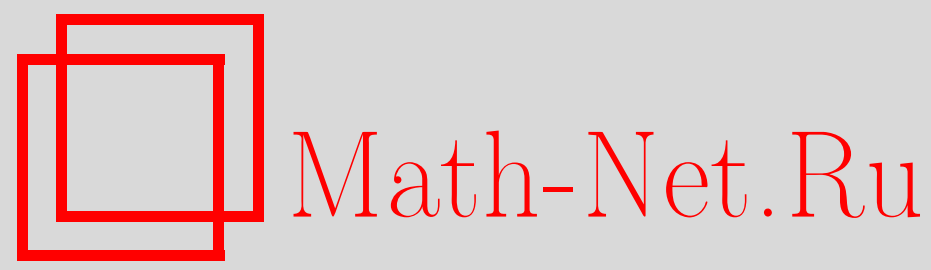

Б. И. Плоткин, Изотипные алгебры, Совр. пробл. матем., 2011, выпуск 15, 40-66

DOI: https://doi.org/10.4213/spm28

Использование Общероссийского математического портала Math-Net.Ru подразумевает, что вы прочитали и согласны с пользовательским соглашением http://www .mathnet.ru/rus/agreement

Параметры загрузки:

IP : 54.205 .225 .156

26 апреля 2023 г., 13:21:21

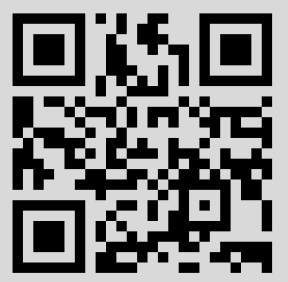




\title{
Изотипные алгебры
}

\author{
Б. И. Плоткин \\ Institute of Mathematics, Hebrew University of Jerusalem, Jerusalem, Israel
}

Посвящается 100-летнему юбилею гениального ученого Анатолия Ивановича Мальцева

\section{1. Общий взгляд}

1.1. Введение. Понятие типа для алгебры, как и для любой другой алгебраической системы пришло из теории моделей и оказалось одним из ключевых понятий (см., например, [1], [2]).

Данная статья посвящена изотипным алгебрам, т.е. алгебрам с одной и той же системой типов. Мы рассматриваем алгебры, принадлежащие некоторому фиксированному многообразию алгебр $\Theta$. Мы подходим к понятию типа с позиций универсальной алгебраической геометрии (UAG). C одной стороны, универсальная алгебраическая геометрия является эквациональной алгебраической геометрией в произвольном многообразии алгебр Ө. Это значит, что алгебраические множества определяются системами уравнений в свободных алгебрах из $\Theta$. С другой стороны, универсальная алгебраическая геометрия распространяется до геометрии логики первого порядка (FOL) в произвольном $\Theta$ (логической геометрии). Это значит, что алгебраические множества определяются произвольными формулами первого порядка, семантически сжатыми в данном $\Theta$. В случае логической геометрии алгебраические множества называются элементарными множествами, а произвольные формулы первого порядка заменяют уравнения (подробности см. в [3]).

В этой статье мы исходим из системы понятий алгебраической логики. В принципе такой подход можно перевести на обычный язык теории моделей. Однако мы убеждены, что применение алгебраической логики делает главные идеи статьи более явными и согласованными.

Мост между логической геометрией и теорией моделей наводится алгебраической логикой с помощью алгебры формул $\Phi=\Phi(X)$, где $X$ - конечное множество переменных. В действительности, $\Phi(X)$ - это некоторое множество формул первого порядка некоторого сорта $X$, свертывающееся определенным образом в алгебру формул. Точное определение алгебры $\Phi=\Phi(X)$ дано в п. 5.5 (см. также [4]-[6]).

Пусть $W=W(X)$ - свободная алгебра в $\Theta$ над $X$. Равенство $w \equiv w^{\prime}$, являющееся элементом алгебры $\Phi(X)$, соответствует равенству $w=w^{\prime}$ в $W(X)$. Таким образом, равенства рассматриваются как нульместные операции (константы) в $\Phi(X)$. Булевы алгебры с равенствами вида $w \equiv w^{\prime}$ и кванторами $\exists x$, действующими по всем $x \in X$, называются расширенными булевыми алгебрами (о списке тождеств см. [3], а также пп. 2.1 и 5.2 настоящей статьи). Алгебра $\Phi(X)$ служит примером расширенной булевой алгебры. Однако в $\Phi(X)$ существуют и другие операции $s_{*}$ (см. ниже).

Как было сказано, универсальная алгебраическая геометрия - это алгебраическая геометрия, ассоциированная с произвольным многообразием алгебр $\Theta$. Если $\Theta=$ Com $-K-$ это многообразие коммутативных ассоциативных алгебр с единицей над полем $P$, то мы получаем классическую алгебраическую геометрию. Одна из основных проблем, относящихся к универсальной алгебраической геометрии, - понять, какая часть богатой геометрии многообразия 
$\Theta=\mathrm{Com}-K$ (т.е. классической алгебраической геометрии) сохраняется в других многообразиях $\Theta$. С другой стороны, новые идеи, относящиеся к UAG и в особенности к логической геометрии, появляются в классической ситуации Сom $-K$.

Важно отметить, что эквациональная алгебраическая геометрия (AG) связана с категорией $\Theta^{0}$ свободных в $\Theta$ алгебр $W=W(X)$ с конечными множествами $X$. Чтобы облегчить интуитивное понимание, нужно упомянуть, что в классическом случае $\Theta^{0}$ является категорией всех полиномиальных алгебр над полем $P$. Роль $\Theta^{0}$ в логической геометрии (LG) играет специальная категория $\operatorname{Hal}_{\Theta}^{0}$, чьи объекты суть алгебры формул $\Phi(X)$ (мы используем обозначение $\mathrm{Hal}$, чтобы напомнить роль П. Халмоша в алгебраической логике). Категории $\Theta^{0}$ и $\mathrm{Hal}_{\Theta}^{0}$ связаны ковариантным функтором

$$
\Theta^{0} \rightarrow \operatorname{Hal}_{\Theta}^{0} .
$$

Этот функтор сопоставляет морфизм $s_{*}: \Phi(X) \rightarrow \Phi(Y)$ в $\mathrm{Hal}_{\Theta}^{0}$ каждому гомоморфизму-морфизму $s: W(X) \rightarrow W(Y)$ в $\Theta^{0}$. Здесь $s_{*}$ является булевым гомоморфизмом, в некотором смысле совместимым с кванторами и тождествами. Этот же $s_{*}$ можно трактовать как операцию в специальной многосортной алгебре Халмоша (подробности см. в параграфе 5).

Сделаем теперь шаг вперед к общей теории. Обозначим через $\Phi^{0}=\Phi^{0}(X)$ подалгебру в $\Phi(X)$, порожденную всеми тождествами в сигнатуре булевых операций и кванторов. Это расширенная булева алгебра, являющаяся подалгеброй расширенной булевой алгебры $\Phi(X)$. Эта подалгебра обычно меньше, чем $\Phi(X)$, так как операции $s_{*}$ не вовлечены в запись элементов из $\Phi^{0}$. Как мы заметим здесь, алгебра $\Phi^{0}(X)$ не может быть определена независимо от алгебры $\Phi(X)$. В свою очередь $\Phi(X)$ определяется посредством алгебраической логики.

ЗАмечание 1.1. Из аксиом алгебр Халмоша (п. 5.3) следует, что если даны $s: W(Y) \rightarrow$ $W(X)$ и равенство $v$ в $\Phi^{0}(Y)$, то $s_{*}(v)$ будет равенством в $\Phi^{0}(X)$. Однако для формулы $v=$ $\exists y v_{0} \in \Phi^{0}(Y)$ формула $s_{*}(v)$ может не лежать в $\Phi^{0}(X)$ (см., например, предложение 4.12).

Рассмотрим теперь происхождение морфизмов и операций $s_{*}$. Причины для введения $s_{*}$ состоят в следующем. Как известно, стандартная алгебраическая логика первого порядка использует бесконечное число переменных. Обозначим это бесконечное множество переменных через $X^{0}$. Предполагая нужды логической геометрии, мы будем иметь дело с системой $Г$ всех конечных подмножеств $X$ в $X^{0}$. В алгебраической логике этот подход ведет к категорям Халмоша и многосортным (т.е. Г-сортным) алгебрам Халмоша. Различные $\Phi(X), X \in \Gamma$, должны быть как-то связаны, что требует введения операций и морфизмов типа $s_{*}$ (подробности см. в параграфе 5, где речь идет об алгебре (категории) $\left.\mathrm{Hal}_{\Theta}(H)\right)$.

Некоторые понятия, используемые нами, можно найти в [3]. Для полноты изложения мы воспроизводим много определений из [3]. Знакомство с работами, посвященными универсальной алгебраической геометрии ([4]-[10] и др.), сделают материал более доступным.

Статья организована следующим образом. В параграфе 1 мы вводим понятие изотипных алгебр и соотносим его с известными понятиями универсальной алгебраической геометрии. Параграф 2 имеет дело с нётеровыми свойствами. В параграфе 3 мы вводим понятие логически отделимой алгебры и приводим примеры изотипных, но не изоморфных алгебр. Параграф 4 посвящен логически совершенным алгебрам. Мы показываем, что всякая группа вкладывается в логически совершенную группу. Кроме того, мы рассматриваем два примера абелевых групп и изучаем их поведение по отношению к логическим свойствам. Параграф 5 призван сделать статью самодостаточной; здесь мы предоставляем читателю необходимые понятия алгебраической логики, в частности, мы определяем алгебру формул $\Phi(X)$, играющую главную роль во всех рассмотрениях. В параграфе 5 находится также список открытых проблем.

Настоящая статья не содержит сложных теорем. Однако она предоставляет новый взгляд и новые проблемы в той области математики, которую можно охарактерезовать как логику 
в алгебре. Что касается предметов исследования, главную роль в статье играет алгебра $H \in \Theta$, которая рассматривается в перспективе ее логических и геометрических инвариантов.

1.2. Алгебра $\operatorname{Bool}(\boldsymbol{W}(\boldsymbol{X}), \boldsymbol{H})$. Пусть $H$ - некоторая алгебра в многообразии $\Theta$. Возьмем свободную в $\Theta$ алгебру $W=W(X)$ с каким-нибудь конечным $X$ и рассмотрим множество $\operatorname{Hom}(W, H)$ как аффинное пространство. Его точки суть гомоморфизмы $\mu: W \rightarrow H$. Если $X=\left\{x_{1}, \ldots, x_{n}\right\}$, то $\operatorname{Hom}(W, H)$ изоморфно $H^{(n)}$ и точка $\mu$ соответствует $n$-ке $\bar{a}=$ $\left(a_{1}, \ldots, a_{n}\right) \in H^{(n)}$.

Булеву алгебру всех подмножеств множества $\operatorname{Hom}(W(X), H)$ обозначим $\operatorname{Bool}(W(X), H)$. Определим действие кванторов $\exists x, x \in X$. Напомним (см. [11]), что если $B$ - булева алгебра, то отображение $\exists: B \rightarrow B$ является экзистенциальным квантором, если выполнены следующие аксиомы:

1) $\exists(0)=0$;

2) $\exists(a) \geqslant a$;

3) $\exists(a \wedge \exists b)=\exists a \wedge \exists b$.

Универсальный квантор $\forall: B \rightarrow B$ определяется двойственно как $\forall(a)=\neg(\exists(\neg a))$.

Пусть теперь $A \in \operatorname{Bool}(W(X), H)$ и $x \in X$. Положим $\mu \in \exists x A$, если найдется такое $\nu \in A$, что $\mu\left(x^{\prime}\right)=\nu\left(x^{\prime}\right)$ для любого $x^{\prime} \in X, x^{\prime} \neq x$. Необходимые условия 1)-3) выполнены и определение экзистенциального квантора прекрасно согласуется с интуицией.

Пусть, далее, $w \equiv w^{\prime}$ - какое-то равенство в алгебре формул $\Phi(X)$. Определим соответствующий элемент алгебры $\operatorname{Bool}(W(X), H)$ как

$$
\operatorname{Val}_{H}^{X}\left(w \equiv w^{\prime}\right)=\left\{\mu: W \rightarrow H \mid\left(w, w^{\prime}\right) \in \operatorname{Ker}(\mu)\right\}
$$

Эти элементы рассматриваются как равенства в $\operatorname{Bool}(W(X), H)$.

Итак, $\operatorname{Bool}(W(X), H)$ определено как расширенная булева алгебра.

Как мы увидим, соответствие $w \equiv w^{\prime} \rightarrow \operatorname{Val}_{H}^{X}\left(w \equiv w^{\prime}\right)$ естественно продолжается до гомоморфизма расширенных булевых алгебр

$$
\operatorname{Val}_{H}^{X}: \Phi(X) \rightarrow \operatorname{Bool}(W(X), H)
$$

Мы рассматриваем также категорию $\operatorname{Hal}_{\Theta}(H)$ всех алгебр вида $\operatorname{Bool}(W(X), H)$ с естественными морфизмами $s_{*}$. Значения и морфизмым в $\operatorname{Hal}_{\Theta}^{0}$ и $\mathrm{Hal}_{\Theta}(H)$ связаны следующей коммутативной диаграммой:

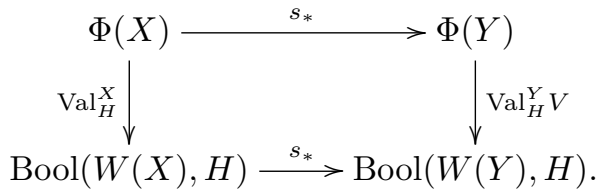

ЗАмЕЧАниЕ 1.2. Существование гомомофизмов $\operatorname{Val}_{H}^{X}$ для любой алгебры $H$ в $\Theta$, удовлетворяющей вышеприведенной диаграмме, было ведущей идеей для определения алгебры формул $\Phi(X)$ и категории таких алгебр формул $\mathrm{Hal}_{\Theta}^{0}$. Здесь очевидно преимущество применения алгебраической логики.

Наша цель - определить систему алгебр формул $\Phi(X)$, где $X$ пробегает конечные подмножества множества $X^{0}$, и определить категорию $\mathrm{Hal}_{\Theta}^{0}$ этих алгебр, таким образом, чтобы условия $(*)$ и $(* *)$ выполнялись для всякой алгебры $H \in \Theta$ и всякого гомоморфизма $s: W(X) \rightarrow W(Y)$.

В действительности, нам нужно определить верхний уровень диаграммы (**) и ее вертикальные стрелки, уже имея заданным нижний уровень диаграммы (это ведет к построениям и условиям, которые будут реализованы в параграфе 5).

Такой подход к определению алгебры $\Phi(X)$ и категории $\mathrm{Hal}_{\Theta}^{0}$ хорошо согласуется с подходом, основанным на применении эквивалентности Линденбаума-Тарского в логике. 
1.3. Логическое ядро точки, типы, изотипные алгебры. Определим понятие логического ядра точки как $\mu: W(X) \rightarrow H$. Таким образом, наряду с (обычным) ядром $\operatorname{Ker}(\mu)$ мы будем рассматривать логическое ядро $\operatorname{LKer}(\mu)$. Это логическое ядро является важным логическим инвариантом точки.

ОПРЕДЕЛЕНИЕ 1.3. Пусть $u \in \Phi(X)$ и $\mu: W(X) \rightarrow H$ - некоторая точка в $\operatorname{Hom}(W(X), H)$. Положим $u \in \operatorname{LKer}(\mu)$, если $\mu \in \operatorname{Val}_{H}^{X}(u)$.

В этом случае мы будем говорить, что $\operatorname{Val}_{H}^{X}(u)$ - значение формуль и в $\operatorname{Bool}(W(X), H)$, а точка $\mu$-решение “уравнения" и в $\operatorname{Hom}(W(X), H)$.

Это определение соответствует обычному индуктивному определению точки, удовлетворяющей формуле (см. [2]).

Имеет место также

$$
\operatorname{Ker}(\mu)=\operatorname{LKer}(\mu) \cap M_{X},
$$

где $M_{X}$ - множество всех равенств $w \equiv w^{\prime}, w, w^{\prime} \in W(X)$.

Покажем, что ядро $\operatorname{LKer}(\mu)$ является ультрафильтром булевой алгебры $\Phi(X)$. Вначале докажем, что оно является фильтром. Пусть $u_{1}, u_{2} \in \operatorname{LKer}(\mu)$. Имеем

$$
\mu \in \operatorname{Val}_{H}^{X}\left(u_{1}\right) \cap \operatorname{Val}_{H}^{X}\left(u_{2}\right)=\operatorname{Val}_{H}^{X}\left(u_{1} \wedge u_{2}\right) .
$$

Значит, $u_{1} \wedge u_{2} \in \operatorname{LKer}(\mu)$. Пусть теперь $u \in \operatorname{LKer}(\mu), v \in \Phi(X)$. Имеем

$$
\mu \in \operatorname{Val}_{H}^{X}(u) \cup \operatorname{Val}_{H}^{X}(v)=\operatorname{Val}_{H}^{X}(u \vee v) .
$$

Таким образом, $u \vee v \in \operatorname{LKer}(\mu)$ и $\operatorname{LKer}(\mu)$ - фильтр.

Пусть теперь $u \in \Phi(X), u \notin \operatorname{LKer}(\mu)$, т.е.

$$
\mu \notin \operatorname{Val}_{H}^{X}(u), \quad \mu \in \neg \operatorname{Val}_{H}^{X}(u)=\operatorname{Val}_{H}^{X}(\neg u) .
$$

Тогда $\neg u \in \operatorname{LKer}(\mu)$ и, значит, $\operatorname{LKer}(\mu)$ - ультрафильтр.

ОПРедЕление 1.4. Всякий ультрафильтр в $\Phi(X)$ назовем $X$-типом. Тип $T$ назовем $X$-типом алгебры $H \in \Theta$, если $T=\operatorname{LKer}(\mu)$ для некоторого $\mu: W(X) \rightarrow H$.

ЗАмечание 1.5. Ср. с определением типа из [2].

Будем говорить, что тип $T$ реализуется в алгебре $H$, если $T$ является $X$-типом алгебры $H$. Обозначим через $S^{X}(H)$ систему всех $X$-типов алгебры $H$. Это важный логический инвариант алгебры $H$.

ОПРедЕлЕНИЕ 1.6. Алгебры $H_{1}$ и $H_{2}$ в $\Theta$ называются изотипными, если для всякого конечного $X$ любой $X$-тип алгебры $H_{1}$ является $X$-типом алгебры $H_{2}$ и наоборот.

Таким образом, алгебры $H_{1}$ и $H_{2}$ изотипны, если

$$
S^{X}\left(H_{1}\right)=S^{X}\left(H_{2}\right) \quad \text { для всех } X .
$$

Далее, заметим, что логическое ядро $\operatorname{LKer}(\mu)$ точки $\mu: W(X) \rightarrow H$ содержит элементарную $X$-теорию алгебры $H$. В самом деле, если $u \in \mathrm{Th}^{X}(H)$, то $\operatorname{Val}_{H}^{X}(u)=\operatorname{Hom}(W(X), H)$. В частности, $\mu \in \operatorname{Val}_{H}^{X}(u)$ и $u \in \operatorname{LKer}(\mu)$. Таким образом, $\operatorname{Th}^{X}(H) \subset \operatorname{LKer}(\mu)$; здесь

$$
\operatorname{Th}^{X}(H)=\left\{u \in \Phi(X) \mid \operatorname{Val}_{H}^{X}(u)=\operatorname{Hom}(W(X), H)\right\} .
$$

Теперь ясно, что если $H_{1}$ и $H_{2}$ изотипны, то

$$
\operatorname{Th}^{X}\left(H_{1}\right)=\operatorname{Th}^{X}\left(H_{2}\right),
$$

где $\operatorname{Th}^{X}(H)$ - элементарная $X$-теория алгебры $H$. 
ОПРЕДЕЛЕНИЕ 1.7. Скажем, что алгебра $H$ является насыщенной, если для всякого $X$ любой ультрафильтр $T$ в $\Phi(X)$, содержащий $\operatorname{Th}^{X}(H)$, реализуется в $H$.

ЗАмечАниЕ 1.8. Определение насыщенной алгебры, используемое в теории моделей, оперирует также с константами. В нашем определении константы уже входят в сигнатуру многообразия $\Theta$.

Из определения следует, что если насыщенные алгебры $H_{1}$ и $H_{2}$ элементарно эквивалентны, т.е. $\operatorname{Th}\left(H_{1}\right)=\operatorname{Th}\left(H_{2}\right)$, то они изотипны. Таким образом, насыщенные алгебры элементарно эквивалентны, если и только если они изотипны. Как мы отметили выше, изотипные алгебры всегда элементарно эквивалентны. Однако понятие изотипности сильнее понятия элементарной эквивалентности. Фактически отношение алгебр "быть изотипными" можно трактовать как обобщение идеи насыщенности алгебр.

В дальнейшем мы рассматриваем логически нётеровы алгебры. Утверждение для таких алгебр также двустороннее: логически нётеровы алгебры $H_{1}$ и $H_{2}$ элементарно эквивалентны, если и только если они изотипны.

Мы будем изучать определение изотипных алгебр с позиции логической геометрии, но сначала обсудим эквациональную алгебраическую геометрию. Заметим, что в алгебраической геометрии также бывает полезно говорить об атомарном ядре, под которым подразумеваются все те равенства $w \equiv w^{\prime}$ и их отрицания $w \not \equiv w^{\prime}$, которые лежат в $\operatorname{LKer}(\mu)$. Обозначим

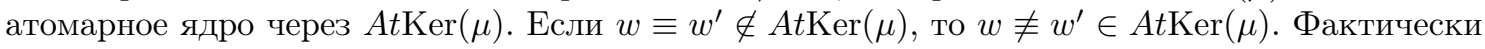
$A t \operatorname{Ker}(\mu)$ является ядром точки $\mu$, представленным в алгебре формул $\Phi(X)$.

Мы рассматриваем также специальное логическое ядро $\operatorname{LKer}^{0}(\mu)$, определенное как

$$
\operatorname{LKer}^{0}(\mu)=\operatorname{LKer}(\mu) \cap \Phi^{0}(X) .
$$

Это ядро является ультрафильтром в алгебре $\Phi^{0}(X)$.

1.4. Основные соответствия Галуа в алгебраической геометрии и логической геометрии. AG- и LG-эквивалентность алгебр. Рассмотрим соответствие Галуа между подмножествами $A$ в $\operatorname{Hom}(W(X), H)$ и системами уравнений $T$ в $W=W(X)$. Эти $T$ можно рассматривать как двуместные отношения в $W$. Для каждого $T$ положим

$$
T_{H}^{\prime}=A=\{\mu: W \rightarrow H \mid T \subset \operatorname{Ker}(\mu)\} .
$$

Для произвольного $A$ положим

$$
A_{H}^{\prime}=T=\bigcap_{\mu \in A} \operatorname{Ker}(\mu)
$$

Мы имеем здесь соответствие Галуа и можем говорить о замыканиях Галуа $A_{H}^{\prime \prime}, T_{H}^{\prime \prime}$. Любое множество $A \subset \operatorname{Hom}(W(X), H)$ вида $A=T_{H}^{\prime}$ замкнуто; назовем его алгебраическим множеством. Любая система уравнений $T$ вида $T=A_{H}^{\prime}$ будет $H$-замкнутой конгруэнцией в $W$.

Сделаем то же самое для логической геометрии, подставляя $\operatorname{LKer}(\mu)$ вместо $\operatorname{Ker}(\mu)$. Здесь $T$ будет произвольным подмножеством в $\Phi=\Phi(X)$. Положим

$$
T_{H}^{L}=A=\{\mu: W \rightarrow H \mid T \subset \operatorname{LKer}(\mu)\}, \quad A_{H}^{L}=T=\bigcap_{\mu \in A} \operatorname{LKer}(\mu) .
$$

Соответствующие замыкания суть $T_{H}^{L L}$ и $A_{H}^{L L}$. Каждое $A$ вида $A=T_{H}^{L}$ назовем элементарным множеством (оно определено и для бесконечного $T$ ). Каждое $T=A_{H}^{L}$ будет $H$-замкнутым фильтром в булевой алгебре $\Phi=\Phi(X)$. Имеем также

$$
T_{H}^{L}=A=\bigcap_{u \in T} \operatorname{Val}_{H}^{X}(u), \quad T=A_{H}^{L}=\left\{u \in \Phi(X) \mid A \subset \operatorname{Val}_{H}^{X}(u)\right\} .
$$


Вспомним, что алгебры $H_{1}$ и $H_{2}$ в $\Theta$ называются геометрически эквивалентными ( $A G$-эквивалентными), если для всякого конечного $X$ и всякого $T$ в $W(X)$ верно

$$
T_{H_{1}}^{\prime \prime}=T_{H_{2}}^{\prime \prime}
$$

(см. [4], [5]), и что $H_{1}$ и $H_{2}$ называются $L G$-эквивалентными, если всегда

$$
T_{H_{1}}^{L L}=T_{H_{2}}^{L L}
$$

для всех $T \subset \Phi(X)$ (см. [3]).

Отметим, что можно, начиная с логического ядра $\operatorname{LKer}^{0}(\mu)$, также установить соответствие Галуа между подмножествами $T$ в $\Phi^{0}(X)$ и множествами точек $A$ в $\operatorname{Hom}(W(X), H)$.

1.5. LG-эквивалентность и изотипные алгебры. Установим соотношения между LG-эквивалентностью и изотипностью алгебр. Пусть $A$ - подмножество в $\operatorname{Hom}(W(X), H)$, состоящее из единственной точки $\mu: W(X) \rightarrow H$. Имеем

$$
T=A_{H}^{L}=\{\mu\}_{H}^{L}=\operatorname{LKer}(\mu) .
$$

Значит, $\operatorname{LKer}(\mu)=\{\mu\}_{H}^{L}$ и $\operatorname{LKer}(\mu)$ является $H$-замкнутым ультрафильтром. Пусть $T$ - ультрафильтр и $T=\operatorname{LKer}(\mu)$. Возьмем $T_{H}^{L}=A_{0}$ и положим $\nu \in A_{0}$. Имеем

$$
\{\nu\}_{H}^{L}=\operatorname{LKer}(\nu) \supset A_{0}^{L}=T=\operatorname{LKer}(\mu) .
$$

Видим, что любые две точки в $A_{0}$ имеют одно и то же логическое ядро. Кроме того, можно заметить, что если $T$ - ультрафильтр в $\Phi(X)$, то $T$ будет $X$-типом для $H$, если и только если, множество $A_{0}=T_{H}^{L}$ непусто.

ОПредЕление 1.9. Определим эквивалентность $\rho=\rho_{H}^{X}$ на множестве $\operatorname{Hom}(W(X), H)$, полагая $\mu \rho \nu$, если и только если $\operatorname{LKer}(\mu)=\operatorname{LKer}(\nu)$.

Рассмотрим фактормножество $\operatorname{Hom}(W(X), H) / \rho=\overline{\operatorname{Hom}}(W(X), H)$. Имеется биекция $\overline{\operatorname{Hom}}(W(X), H) \rightarrow S^{X}(H)$.

Заметим, что любой класс эквивалентности $\rho$ является элементарным множеством, заданным типом $\operatorname{LKer}(\mu)=T$, где $\mu$ принадлежит этому классу эквивалентности. Согласно [5] каждое элементарное множество инвариантно относительно действия группы автоморфизмов $\operatorname{Aut}(H)$. Следовательно, если $\mu$ и $\nu$ сопряжены некоторым автоморфизмом $\sigma \in \operatorname{Aut}(H)$, то $\mu \rho \nu$. Вспомним, что действие группы $\operatorname{Aut}(H)$ в $\operatorname{Hom}(W, H)$ определяется сдвигами $\mu \rightarrow \mu \sigma$. При определенных условиях обратное также верно. В этих случаях классы эквивалентности $\rho$ суть в точности орбиты действия группы $\operatorname{Aut}(H)$.

Следующая теорема основная в статье.

ТеОрема 1.10. Алгебры $H_{1}$ и $H_{2}$ являются $L G$-эквивалентными, если и только если они изотипны.

ДокАЗАтЕльСтво. Пусть $H_{1}$ и $H_{2}$ - некоторые LG-эквивалентные алгебры. Это означает, что для всякого конечного $X$ и всякого множества $T$ формул из $\Phi(X)$ данное $T$ будет $H_{1}$-замкнутым, если и только если $T$ является $H_{2}$-замкнутым.

Возьмем теперь какой-нибудь ультрафильтр $T$ в $\Phi(X)$, и пусть $T$ будет $X$-типом над $H_{1}$. Этот $T$ является $H_{1}$-замкнутым и, следовательно, $H_{2}$-замкнутым. Здесь $T_{H_{2}}^{L}$ непусто и, значит, $T$ есть $X$-тип над $H_{2}$. Переход от $H_{2}$ к $H_{1}$ осуществляется подобным же образом. Следовательно, $H_{1}$ и $H_{2}$ изотипны.

Пусть, далее, $H_{1}$ и $H_{2}$ изотипны. Это означает, в частности, что если $T=\operatorname{LKer}(\mu)$ для $\mu: W(X) \rightarrow H_{1}$, то также $T=\operatorname{LKer}(\nu)$ для некоторого $\nu: W(X) \rightarrow H_{2}$. То же самое верно и при переходе от $H_{2}$ к $H_{1}$. Таким образом, $T$ одновременно $H_{1}$ - и $H_{2}$-замкнуто. 
Пусть теперь $T$ - какой-нибудь $H_{1}$-замкнутый фильтр в $\Phi(X)$. Мы хотим проверить, что $T$ является $H_{2}$-замкнутым. Возьмем $A=T_{H_{1}}^{L}$. Тогда

$$
T=A_{H_{1}}^{L}=\bigcap_{\mu \in A} \operatorname{LKer}(\mu) .
$$

Это значит, что $u \in T$ равносильно $A \subset \operatorname{Val}_{H_{1}}^{X}(u)$.

Покажем, что пересечение $H$-замкнутых фильтров также $H$-замкнуто. Как мы знаем, любой $H_{1}$-замкнутый фильтр вида $\operatorname{LKer}(\mu)$ является $H_{2}$-замкнутым. Таким образом, $T=$ $\bigcap_{\mu \in A} \operatorname{LKer}(\mu)$ является $H_{2}$-замкнутым фильтром. Подобным же образом $H_{2}$-замкнутость множества $T$ влечет его $H_{1}$-замкнутость. Это верно для любого $X$. Значит, $H_{1}$ и $H_{2}$ являются LG-эквивалентными.

Остается проверить, что пересечение $T=\bigcap_{\alpha} T_{\alpha}$ по всем $H$-замкнутым $T_{\alpha}, \alpha \in I$, где $I-$ некоторое множество индексов, также $H$-замкнуто.

Возьмем $T_{\alpha}=\left(A_{\alpha}\right)_{H}^{L}$ и проверим, что $\bigcap_{\alpha}\left(A_{\alpha}\right)_{H}^{L}=\left(\bigcup_{\alpha} A_{\alpha}\right)_{H}^{L}$. Пусть $u \in \bigcap_{\alpha} T_{\alpha}=T$. Включения $u \in T_{\alpha}$ и $A_{\alpha} \subset \operatorname{Val}_{H}^{X}(u)$ всегда выполнены. Следовательно, $\bigcup_{\alpha} A_{\alpha} \subset \operatorname{Val}_{H}^{X}(u)$ и, значит, $u \in\left(\bigcup_{\alpha} A_{\alpha}\right)_{H}^{L}$.

Пусть $u \in\left(\bigcup_{\alpha} A_{\alpha}\right)_{H}^{L}$. Тогда $\bigcup_{\alpha} A_{\alpha} \subset \operatorname{Val}_{H}^{X}(u)$. Включения $A_{\alpha} \subset \operatorname{Val}_{H}^{X}(u)$ и $u \in\left(A_{\alpha}\right)_{H}^{L}=T_{\alpha}$ всегда выполнены. Следовательно, $u \in T$.

Из данной теоремы следует, что если алгебры $H_{1}$ и $H_{2}$ являются LG-эквивалентными, то они элементарно эквивалентны (см. [3]). Кроме того, если $H_{1}$ и $H_{2}$ изотипны, то категории элементарных множеств $L K_{\Theta}\left(H_{1}\right)$ и $L K_{\Theta}\left(H_{2}\right)$ (см. [3]) изоморфны (также см. параграф 5).

ЗАмЕчАниЕ 1.11. Наше определение типа соответствует понятию полного типа в теории моделей. Полный тип - это $H$-замкнутый ультрафильтр, заданный одной точкой. Однако, произвольное $H$-замкнутое множество задается множеством точек. В этом случае также существуют связи с общей теорией типов в теории моделей. В частности, $H$-замыкание произвольного типа всегда будет пересечением полных типов.

1.6. Инфинитарная логика. Сделаем несколько замечаний, касающихся связей с инфинитарной логикой. Начнем с квазитождеств. Возьмем некоторое двуместное отношение $T$ на $W=W(X)$. Рассмотрим формулу (квазитождество)

$$
\left(\bigwedge_{\left(w, w^{\prime}\right) \in T}\left(w \equiv w^{\prime}\right)\right) \rightarrow\left(w_{0} \equiv w_{0}^{\prime}\right) .
$$

Если $T$ бесконечно, эта формула будет инфинитарным квазитождеством.

Пусть, далее, $H$ - какая-то алгебра в $\Theta$; рассмотрим $T_{H}^{\prime \prime}$. В [12] доказано, что $\left(w_{0}, w_{0}^{\prime}\right) \in T_{H}^{\prime \prime}$, если и только если квазитождество $(\star)$ выполнено в $H$.

Исходя теперь из $T \subset \Phi(X)$ рассмотрим формулу

$$
\left(\bigwedge_{u \in T} u\right) \rightarrow v
$$

где $v \in \Phi(X)$. Формула $(\star \star)$ инфинитарна, если множество $T$ бесконечно. Будем писать для краткости $T \rightarrow v$. Эта формула выполняется в $H$, если и только если $v \in T_{H}^{L L}$ (см. [6]). Обозначим через $\operatorname{Im}(\mathrm{Th})(H)$ множество всех формул вида $T \rightarrow v$, выполненных в $H$. Оно является импликативной теорией алгебры $H$. Можно показать, что алгебры $H_{1}$ и $H_{2}$ являются LG-эквивалентными (изотипными) тогда и только тогда, когда их импликативные теории совпадают, т.е. $\operatorname{Im}(\mathrm{Th})\left(H_{1}\right)=\operatorname{Im}(\mathrm{Th})\left(H_{2}\right)$ (см. [3]). Присутствие здесь инфинитарных формул обусловлено задачами универсальной алгебраической геометрии. Эти формулы, конечно, хорошо известны и в теории моделей. В частности, они участвуют в теории абстрактных элементарных классов (АЕС-классов) моделей (см. [13]). 
1.7. Соответствие Галуа и морфизмы в $\mathbf{H a l}_{\Theta}^{0}$. Установим соотношение между соответствием Галуа и морфизмами в категории $\operatorname{Hal}_{\Theta}^{0}$. Пусть даны какие-то $s: W(X) \rightarrow W(Y)$ и $s_{*}: \Phi(X) \rightarrow \Phi(Y)$. Для любого $u \in \Phi(X)$ имеем

$$
\operatorname{Val}_{H}^{Y}\left(s_{*} u\right)=s_{*} \operatorname{Val}_{H}^{X}(u) .
$$

Здесь $\mu \in s_{*} \operatorname{Val}_{H}^{X}(u)$, если $\mu s \in \operatorname{Val}_{H}^{X}(u)$.

Для $T \subset \Phi(X)$ обозначим через $s_{*} T$ подмножество в $\Phi(Y)$, состоящее из $s_{*} u, u \in T$. Имеем

$$
\left(s_{*} T\right)_{H}^{L}=s_{*} T_{H}^{L}
$$

(см. [5]).

Таким образом, если $A=T_{H}^{L}$ - элементарное множество в $\operatorname{Hom}(W(X), H)$, то $s_{*} A$ - элементарное множество в $\operatorname{Hom}(W(Y), H)$. Как обычно, $\mu \in s_{*} A$, если $\mu s \in A$.

Свяжем теперь $X$ - и $Y$-типы над $H$ для данных $s: W(X) \rightarrow W(Y)$ и $s_{*}: \Phi(X) \rightarrow \Phi(Y)$. Для каждой точки $\nu: W(X) \rightarrow H$ обозначим через $\operatorname{LKer}^{X}(\nu)$ ее логическое ядро, помня, что это ядро вычислено в $\Phi(X)$. Проверим, что

$$
s_{*} \operatorname{LKer}^{X}(\mu s) \subset \operatorname{LKer}^{Y}(\mu)
$$

для $\mu: W(Y) \rightarrow H$.

Пусть $u \in \Phi(X)$, и пусть $u \in \operatorname{LKer}^{X}(\mu s)$. Это дает $\mu s \in \operatorname{Val}_{H}^{X}(u)$. Таким образом, $\mu \in$ $s_{*} \operatorname{Val}_{H}^{X}(u)=\operatorname{Val}_{H}^{Y}\left(s_{*} u\right)$, т.е. $s_{*} u \in \operatorname{LKer}^{Y}(\mu)$. Включение проверено.

Применим снова $L$-переход. Имеем

$$
\left(\operatorname{LKer}^{Y}(\mu)\right)_{H}^{L}=B \subset\left(s_{*} \operatorname{LKer}^{X}(\mu s)\right)_{H}^{L}=s_{*}\left(\operatorname{LKer}^{X}(\mu s)\right)_{H}^{L}=s_{*} A .
$$

Здесь $B$ - замыкание точки $\mu, A$ - замыкание точки $\mu s$, а также $B \subset \operatorname{Hom}(W(Y), H)$, $A \subset \operatorname{Hom}(W(X), H), s_{*} A \subset \operatorname{Hom}(W(Y), H)$ и $B \subset s_{*} A ; B$ и $A$ - минимальные элементарные множества (т.е. не содержащие других элементарных множеств), тогда как множество $s_{*} A$ не обязательно минимально.

1.8. Отношения $\boldsymbol{\rho}$ и $\boldsymbol{\tau}$. Наряду с отношением $\rho$ рассмотрим отношения $\rho_{0}$ и $\tau$ на данном множестве $\operatorname{Hom}(W(X), H)$. Отношение $\rho_{0}$ задается разложением множества $\operatorname{Hom}(W(X), H)$ на орбиты группы $\operatorname{Aut}(H)$. Включение $\rho_{0} \subset \rho$ выполнено всегда, но нас интересует ситуация $\rho_{0}=\rho$.

Пусть $X=\left\{x_{1}, \ldots, x_{n}\right\}$, и пусть

$$
\left(\mu\left(x_{1}\right), \ldots, \mu\left(x_{n}\right)\right)=\bar{a}=\left(a_{1}, \ldots, a_{n}\right), \quad\left(\nu\left(x_{1}\right), \ldots, \nu\left(x_{n}\right)\right)=\bar{b}=\left(b_{1}, \ldots, b_{n}\right)
$$

для точек $\mu$ и $\nu$. Обозначим через $A$ и $B$ подалгебры в $H$, порожденные всеми $a_{1}, \ldots, a_{n}$ и всеми $b_{1}, \ldots, b_{n}$ соответственно. Положим $\mu \tau \nu$, если и только если соответствия $a_{i} \rightarrow b_{i}$ задают изоморфизм $\eta: A \rightarrow B$. Имеем $\rho_{0} \subset \tau$. Имеет место следующая теорема.

Теорема 20. Условие $\mu \tau \nu$ выполняется тогда и только тогда, когда $\operatorname{At\operatorname {Ker}}(\mu)=\operatorname{At} \operatorname{Ker}(\nu)$ в $\Phi(X)$, или, что то же самое, $\operatorname{Ker}(\mu)=\operatorname{Ker}(\nu)$ в $W(X)$.

ДокАЗАТЕЛЬСтво. Пусть выполнено $\mu \tau \nu$ и задан соответствующий изоморфизм $\eta: A \rightarrow B$. Возьмем $w \equiv w^{\prime} \in A t \operatorname{Ker}(\mu)$. Имеем $w^{\mu}=w^{\prime \mu}$ в $A$ и $w^{\mu \eta}=w^{\prime \mu \eta}$ в $B$. Равенство $w^{\nu}=w^{\prime \nu}$ выполнено в $B$ и, значит, $w \equiv w^{\prime} \in A t \operatorname{Ker}(\nu)$. Подобным же образом, если $w \not \equiv w^{\prime} \in A t \operatorname{Ker}(\mu)$, то $w \not \equiv w^{\prime} \in A t \operatorname{Ker}(\nu)$, так что $A t \operatorname{Ker}(\mu) \subset A t \operatorname{Ker}(\nu)$. Обратное включение доказывается аналогично.

Проверим обратное утверждение. Рассмотрим гомоморфизмы

$$
\alpha: W(X) \rightarrow A, \quad \beta: W(X) \rightarrow B,
$$


где $X=\left\{x_{1}, \ldots, x_{n}\right\}$ и $\alpha\left(x_{i}\right)=a_{i}=\mu\left(x_{i}\right), \beta\left(x_{i}\right)=b_{i}=\nu\left(x_{i}\right)$. Пусть $w\left(x_{1}, \ldots, x_{n}\right)=$ $w^{\prime}\left(x_{1}, \ldots, x_{n}\right)$ лежат в ядре $\operatorname{Ker}(\alpha)$. Тогда $w \equiv w^{\prime} \in A t \operatorname{Ker}(\mu)=A t \operatorname{Ker}(\nu)$. Следовательно, $w \equiv w^{\prime} \in \operatorname{Ker}(\beta)$. Точнее, $w \equiv w^{\prime} \in \operatorname{Ker}(\alpha)$ равносильно $w \equiv w^{\prime} \in \operatorname{Ker}(\beta)$, т.е. $\operatorname{Ker}(\alpha)=\operatorname{Ker}(\beta)$. Получаем изоморфизм $A \rightarrow B$, индуцированный отображением $a_{i} \rightarrow b_{i}$.

Ясно, что $\operatorname{LKer}(\mu)=\operatorname{LKer}(\nu)$ влечет $A t \operatorname{Ker}(\mu)=A t \operatorname{Ker}(\nu)$; это означает, что $\rho \subset \tau$. Получаем $\rho_{0} \subset \rho \subset \tau$, и если $\tau=\rho_{0}$ для данных $X$ и $H$, то $\rho=\rho_{0}$.

Покажем, что всякий класс эквивалентности $\tau$ является элементарным множеством. Пусть $T$ - произвольная конгруэнция алгебры $W=W(X)$. Рассмотрим множество формул в $\Phi(X)$, заданное равенством $T^{\prime}=T_{1}^{\prime} \cup T_{2}^{\prime}$, где $T_{1}^{\prime}=\left\{w \equiv w^{\prime} \mid\left(w, w^{\prime}\right) \in T\right\}$ и $T_{2}^{\prime}=\left\{w \not \equiv w^{\prime} \mid\left(w, w^{\prime}\right) \notin\right.$ $T\}$. Легко понять, что точка $\mu: W(X) \rightarrow H$ удовлетворяет множеству формул $T^{\prime}$, т.е. $\mu \in$ $\left(T^{\prime}\right)_{H}^{L}$, если и только если $\operatorname{Ker}(\mu)=T$. Значит, класс эквивалентности $\tau$, содержащий точку $\mu$, является элементарным множеством, определенным множеством формул $T^{\prime}$ с $\operatorname{Ker}(\mu)=T$. (Мы всегда будем использовать это замечание, имея дело с отношением $\tau$.)

Заметим, что основной проблемой в этой статье будет нахождение условий на алгебру $H$, представляющих изоморфизм $\eta: A \rightarrow B$, реализующийся некоторым автоморфизмом алгебры $H$. В этом случае имеем $\tau=\rho_{0}$.

1.9. Изотипность точек. Наряду с понятием изотипных алгебр введем понятие изотипных точек над алгебрами. Скажем, что две $X$-точки $\mu, \nu: W(X) \rightarrow H$ uзотипны, если $\operatorname{LKer}(\mu)=\operatorname{LKer}(\nu)$ (это значит, что выполнено $\mu \rho \nu)$.

Это понятие ведет к определению логической изотипности также для элементов алгебры $H$. Скажем, что элементы $a_{1}$ и $a_{2}$ алгебры $H$ изотипны, если соответствующие точки $\mu, \nu \in \operatorname{Hom}(W(x), H)$ с $\mu(x)=a_{1}$ и $\nu(x)=a_{2}$ изотипны.

В действительности, понятие изотипных элементов алгебры $H$ имеет следующий смысл. Будем исходить из формулы или множества формул $T$. Пусть элементы $a_{1}$ и $a_{2}$ из $H$ с соответствующими точками $\mu, \nu \in \operatorname{Hom}(W(x), H)$ изотипны. Тогда точки $\mu$ и $\nu$ изотипны, и пусть $T$ принадлежит $\operatorname{LKer}(\mu)$. Тогда $T$ тоже принадлежит $\operatorname{LKer}(\nu)$. Итак, обе точки удовлетворяют $T$, и пусть формулы из $T$ описывают некоторое алгебраическое свойство элементов из $H$. Изотипность элементов $a_{1}$ и $a_{2}$ означает, что оба этих элемента удовлетворяют данному алгебраическому свойству. Например, если $g$ и $g^{\prime}$ изотипны и $g$ имеет конечный порядок $n$, то $g^{\prime}$ имеет тот же самый порядок. Здесь $T$ состоит из формулы $x^{n} \equiv 1$.

В качестве другого примера рассмотрим энгелевские элементы и ниль-элементы групп. Напомним, что элемент $g \in H$ называется $n$-энгелевским элементом, если найдется такое $n=n(g)$, что $[a, g, \ldots, g]=1$ для всякого $a \in H$. Здесь $[a, g, \ldots, g]$ обозначает составной коммутатор, в котором $g$ повторено $n$ раз. Таким образом, точка $\mu$ с $\mu(x)=a, \mu(y)=g$ удовлетворяет формуле $\forall x([x, y, \ldots, y]=1)$ тогда и только тогда, когда $g$ является $n$-энгелевским элементом. Эта формула есть $T$.

Далее считаем $\mu, \nu: W(x, y) \rightarrow H, \mu(x)=a, \mu(y)=g, \nu(x)=a^{\prime}, \nu(y)=g^{\prime}$. Пусть $\mu$ и $\nu$ изотипны (т.е. $g$ и $g^{\prime}$ изотипны), и пусть эдемент $g$ является $n$-энгелевским. Тогда $g^{\prime}$ также будет энгелевским. Эти наблюдения показывают, что если $H$ - нётерова группа, а $g$ принадлежит ее нильпотентному радикалу, то $g^{\prime}$ тоже принадлежит ему (см. [14] и [15]). Аналогичный факт имеет место для разрешимых радикалов нётеровой группы для соответствующего $T$ и изотипных $g$ и $g^{\prime}$ (см. [16]).

Перейдем к ниль-элементам. Элемент $g$ группы $G$ называется ниль-элементом, если для всякого $a \in G$ найдется такое $n=n(a, g)$, что $[a, g, \ldots, g]=1$, где $g$ взято $n$ раз. Свойство быть ниль-элементом не выразимо формулой первого порядка, поскольку определение использует квантор вида $\exists n$ по натуральным $n$.

Можно улучшить это положение следующим образом. Фиксируем в группе $G$ два элемента $g$ и $g^{\prime}$ и рассмотрим все возможные пары $(a, g)$ и $\left(a^{\prime}, g^{\prime}\right)$, где $a$ и $a^{\prime}$ взаимно однозначно соотнесены. Предположим, что соответствующие $\mu, \nu: W(x, y) \rightarrow H$ изотипны. Допустим теперь, что $g$ - ниль-элемент. Тогда пара $(a, g)$ удовлетворяет равенству $[x, y, \ldots, y]=1$. Изотипность 
точек $\mu, \nu$ влечет, что пара $\left(a^{\prime}, g^{\prime}\right)$ удовлетворяет этому же самому равенству. Это верно для любых подходящих $a$ и $a^{\prime}$, значит, $g^{\prime}$ - тоже ниль-элемент.

Пусть теперь $G$ - разрешимая группа (или, более общим образом, радикальная группа; см. [17]), а $g$ и $g^{\prime}$ - ее изотипные элементы. Тогда если один из них принадлежит некоторому локально нильпотентному радикалу, то и второй тоже принадлежит ему. Это следует из предыдущих замечаний, а также из [17]. Также можно рассматривать различные другие радикалы в других группах. На этом пути возникает множество естественных проблем.

\section{2. Логическая нётеровость}

2.1. Определения и проблемы. Пусть $H$ - некоторая алгебра из многообразия $\Theta$. Рассмотрим три условия нётеровости для $H$.

1. Алгебра $H$ называется логически нётеровой, если для всякого конечного множества формул $X$ произвольное элементарное множество $A \subset \operatorname{Hom}(W(X), H)$ является конечно определимым. Это означает, что если $A=T_{H}^{L}$, то найдется такое конечное множество $T_{0}$ (не обязательно подмножество в $T)$, что $A=\left(T_{0}\right)_{H}^{L}$.

2. Алгебра $H$ называется строго логически нётеровой, если для всякого конечного $X$ и произвольного элементарного множества $A=T_{H}^{L} \subset \operatorname{Hom}(W(X), H)$ найдется такое конечное множество $T_{0} \subset T$, что $A=\left(T_{0}\right)_{H}^{L}$.

3. Алгебра $H$ называется слабо логически нётеровой, если для всякого $T \rightarrow v$, выполненного в $H$, найдется такое конечное подмножество $T_{0} \subset T$ (возможно, зависящее от $v$ ), что $T_{0} \rightarrow v$ выполнено в $H$.

Для каждого из этих условий нётеровости изотипность алгебр $H_{1}$ и $H_{2}$ равносильна их элементарной эквивалентности.

Очевидно, всякая конечная алгебра логически нётерова в любом смысле. В теории моделей приводятся примеры бесконечных логически нётеровых алгебр (см. предложение 2.4).

Нам будет полезно следующее замечание. Все элементарные множества в данном множестве $\operatorname{Hom}(W(X), H)$ образуют подрешетку решетки всех подмножеств. Двойственно, имеем подрешетку всех $H$-замкнутых фильтров в $\Phi(X)$. Строгая нётеровость алгебры $H$ равносильна условию нётеровости соответствующей решетки фильтров и, что то же самое, условию артиновости решетки элементарных множеств для конечного $X$.

Принципиальная проблема, относящаяся к теории моделей, состоит в следующем.

ПроБЛЕма 2.1. Развить общий подход построения логически нётеровых алгебр $H$ в различных многообразиях $\Theta$.

Здесь $\Theta$ может быть многообразием групп, многообразием абелевых групп, многообразием коммутативных ассоциативных алгебр. Например, имеется проблема описания логической нётеровых абелевых групп.

Сделаем несколько замечаний об алгебрах $H$ с конечным множеством типов $S^{X}(H)$ для любого $X$. Из определений следует, что если множество $S^{X}(H)$ конечно, то имеется и конечное число различных $H$-замкнутых фильтров в $\Phi(X)$. Также имеется конечное число элементарных множеств в $\operatorname{Hom}(W(X), H)$. Это дает артиновость и нётеровость соответствующих решеток и строгую нётеровость алгебры $H$. Понятно, что если имеется конечное число $\operatorname{Aut}(H)$-орбит в $\operatorname{Hom}(W(X), H)$, то и множество $S^{X}(H)$ конечно.

2.2. Автоморфная финитарность алгебр. В этом пункте мы рассматриваем проблемy 2.1.

ОПРЕДЕЛЕНИЕ 2.2. Алгебра $H$ называется автоморфно $(\operatorname{Aut}(H))$-финитарной, если при любом конечном $X$ существует лишь конечное число $\operatorname{Aut}(H)$-орбит в $\operatorname{Hom}(W(X), H)$. 
Если $H$ является $\operatorname{Aut}(H)$-финитарным, то существует лишь конечное число типов на $H$ и, значит, лишь конечное число элементарных классов. Поэтому алгебра $H$ строго логически нётерова. Таким образом, возникает вопрос, будет ли алгебра $H$ автоморфно финитарной.

Поставим эту проблему в частном случае групп.

ПроБлемА 2.3. Развить общие методы построения бесконечных автоморфно финитарных групп.

Рассмотрим один пример.

ПредЛОЖЕНИЕ 2.4. Пусть $H$ - бесконечная абелева группа, удовлетворяющая тождеству $x^{p} \equiv 1$ с простым $p$. Такая группа $\operatorname{Aut}(H)$-финитарна.

ЗАмечАниЕ 2.5. Это предложение не неожиданно с точки зрения хорошо известного теоретико-модельного результата, который доказал Рыль-Нарджевский (см. [18], [2]).

ДокАзАтЕльство. Работаем с многообразием $\Theta$ всех абелевых групп, удовлетворяющих тождеству $x^{p} \equiv 1$, где $p$ простое. Фиксируем $X=\left\{x_{1}, \ldots, x_{n}\right\}$, и пусть $W=W(X)-$ свободная в $\Theta$ группа над $X$. Эта группа конечна. Пусть $T$ - подгруппа группы $W$.

Возьмем формулу (в аддитивной записи) $u=\left(\bigwedge_{u_{i} \in T}\left(u_{i} \equiv 0\right)\right) \wedge\left(\bigwedge_{v_{i} \notin T}\left(v_{i} \not \equiv 0\right)\right)$. Обозначим $\operatorname{Val}_{H}^{X}(u)=V$. Очевидно, точка $\mu: W \rightarrow H$ принадлежит $V \subset \operatorname{Hom}(W(X), H)$ тогда и только тогда, когда $\operatorname{Ker}(\mu)=T$.

Пусть теперь $\mu$ и $\nu$ - две точки в $V$, а $A$ и $B$ - их образы в $H$. Тогда $A=\left\langle a_{1}, \ldots, a_{n}\right\rangle$, $B=\left\langle b_{1}, \ldots, b_{n}\right\rangle$. Поскольку $\operatorname{Ker}(\mu)=\operatorname{Ker}(\nu)$, имеем следующую коммутативную диаграмму:

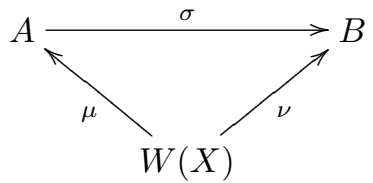

Здесь $\sigma$ - изоморфизм, $\sigma\left(a_{i}\right)=b_{i}$ и $\mu \sigma=\nu$. Подгруппы $A$ и $B$ имеют дополнения в $H$, т.е. $A \oplus A^{\prime}=B \oplus B^{\prime}=H$. Поскольку $H$ бесконечна, $A^{\prime}$ и $B^{\prime}$ бесконечны и изоморфны. Это означает, что изоморфизм $\sigma: A \rightarrow B$ расширяется до автоморфизма $\sigma \in \operatorname{Aut}(H)$. Следовательно, $\mu$ и $\nu$ сопряжены посредством этого автоморфизма. Кроме того, последовательности $\left(a_{1}, \ldots, a_{n}\right)$, $\left(b_{1}, \ldots, b_{n}\right)$ тоже сопряжены посредством автоморфизма $\sigma$. Это означает, что множество $V$ принадлежит $\operatorname{Aut}(H)$-орбите, заданной каждой из точек $\mu$ и $\nu$. д другой стороны, так как $V$ - элементарное множество, точечная орбита содержится в $V$. Следовательно, $V$ равно орбите, содержащей точку $\mu$.

Всякая орбита, заданная точкой $\mu: W \rightarrow H$, имеет такой вид. В самом деле, возьмем $T=\operatorname{Ker}(\mu)$, и пусть формула $u$ построена посредством $T$.

Тогда $V=\operatorname{Val}_{H}^{X}(u)$ будет $\operatorname{Aut}(H)$-орбитой, заданной точкой $\mu$.

Поскольку существует лишь конечное множество различных $T$ и $u$, имеем конечное множество орбит. Более того, все они суть одноопределенные (определяемые одной формулой) элементарные множества. Группа $H$ является бесконечной строго логически нётеревой группой.

Заметим, что в этом доказательстве используется представление точки $\mu \in H^{(n)}$ как точки-гомоморфизма $\mu: W \rightarrow H$.

Схожая ситуация имеет место для бесконечномерных векторных пространств над произвольным конечным полем. Если это поле бесконечно, то описание орбит остается тем же самым, но число орбит становится бесконечным. Различные другие примеры бесконечных автоморфно финитарных групп и строго логически нётеровых групп были предложены мне А. Ольшанским (алгебраический подход) и Б. Зильбером (теоретико-модельный подход). Группы в их конструкциях далеко не абелевы (см. также параграф 4.3). 
В этой связи возникает проблема о существовании бесконечных нётеровых групп, которые также логически нётеровы. Нётеровы полициклические группы и их конечные расширения не подходят. Бесконечные циклические группы также не являются логически нётеровыми.

Закончим этот параграф одним полезным замечанием.

Предположим, что $H_{1}$ и $H_{2}$ изотипны и $H_{1}$ строго логически нётерова. Тогда $H_{2}$ тоже строго логически нётерова.

В самом деле, пусть $H_{1}$ и $H_{2}$ изотипны и $H_{1}$ строго логически нётерова. Тогда для $T \subset \Phi(X)$ и некоторой конечной части $T_{0} \subset T$ имеем $T_{H_{2}}^{L L}=T_{H_{1}}^{L L}=T_{0}^{L L} H_{1}=T_{0}^{L L} H_{2}$ и, значит, $T_{H_{2}}^{L L}=T_{0}^{L L} H_{2}$. Следовательно, $H_{2}$ строго логически нётерова.

\section{3. Изотипность и изоморфизм}

3.1. Отделимые алгебры. Другой общей проблемой является изучение отношений между свойством изотипности и изоморфизмом алгебр. Прежде всего заметим, что существуют неизоморфные изотипные алгебры. Даже в случае полей существуют примеры такого рода (мы приведем примеры изотипных, но не изоморфных алгебр в п. 3.2).

ОПРЕДЕлЕниЕ 3.1. Алгебра $H \in \Theta$ называется отделимой в $\Theta$, если каждая алгебра $H^{\prime} \in \Theta$, изотипная с $H$, изоморфна $H$.

ЗАмЕчАНИЕ 3.2. Иногда стоит изменить определение 3.1 и рассматривать только конечно порожденные $H^{\prime}$.

Проблема 3.3. Для каких $\Theta$ всякая свободная в $\Theta$ алгебра $W=W(X)$ с конечным $X$ отделима?

Другими словами, когда всякая свободная в $\Theta$ алгебра $W=W(X)$ с конечным $X$ может быть различима в $\Theta$ средствами логики типов (т.е. LG-логики)?

Эта проблема поставлена для произвольного многообразия $\Theta$, но здесь более всего мы интересуемся многообразием групп $\Theta=\operatorname{Grp}$ и многообразием $\Theta=\operatorname{Com}-K$ коммутативных и ассоциативных алгебр над полем $P$.

3. Села (не опубликовано) показал, что свободные некоммутативные группы $F(X)$ и $F(Y)$ изотипны, если и только если они изоморфны.

\section{2. Примеры неизоморфных изотипных алгебр.}

ПРЕДЛОЖЕНИЕ 3.4. Пусть алгебры $H_{1}$ и $H_{2}$ - бесконечномерные векторные пространства над полем Р. Тогда они изотипны.

ДокАЗАТЕЛьство. Используемый нами метод приложим также к другим случаям. Исходим из многообразия $\Theta$ векторных пространств над $P$. Возьмем $X=\left\{x_{1}, \ldots, x_{n}\right\}$, и пусть $W=W(X)$ - соответствующий свободный объект, т.е. произвольное линейное пространство размерности $n$. Рассмотрим точки $\mu: W \rightarrow H_{1}$ и $\nu: W(X) \rightarrow H_{2}$. Последовательность $\bar{a}=\left(a_{1}, \ldots, a_{n}\right), a_{i}=\mu\left(x_{i}\right)$, соответствует точке $\mu$. Аналогично, имеем $\bar{b}=\left(b_{1}, \ldots, b_{n}\right)$ для $\nu$. Обозначим $A=\left\langle a_{1}, \ldots, a_{n}\right\rangle$ и $B=\left\langle b_{1}, \ldots, b_{n}\right\rangle$. Точки $\mu$ и $\nu$ назовем изоморбными, если найдется такой изоморфизм $\alpha: A \rightarrow B$, что $\alpha\left(a_{i}\right)=b_{i}$. Пишем $\alpha \mu=\nu$. Имеем также $\mu=\alpha^{-1} \nu$. Если $H_{1}=H_{2}$, то изоморфизм точек $\mu$ и $\nu$ означает, что $\mu$ и $\nu$ удовлетворяют отношению $\tau$.

Наряду со свободной алгеброй $W=W(X)$ рассмотрим алгебру формул $\Phi=\Phi(X)$. Формулу $u \in \Phi$ назовем правильной, если для любых изоморфных $\mu$ и $\nu$ включение $\mu \in \operatorname{Val}_{H_{1}}(u)$, т.е. $u \in \operatorname{LKer}(\mu)$, выполнено тогда и только тогда, когда $u \in \operatorname{LKer}(\nu)$, т.е. $\nu \in \operatorname{Val}_{H_{2}}(u)$.

Мы намерены проверить, что в нашем случае любая формула $u$ правильна. Легко видеть, что: для произвольного $\Theta$ все равенства $w \equiv w^{\prime}$ правильны; если формула $u$ правильна, то ее отрицание $\neg u$ правильно; если обе формулы $u_{1}$ и $u_{2}$ правильны, то $u_{1} \vee u_{2}$ и $u_{1} \wedge u_{2}$ тоже правильны. 
Между тем, импликация "если формула $u$ правильна, то такова и формула $\exists x_{i} u$ при всяком $x_{i}$ ", видимо, не справедлива для произвольного Ө. Здесь возникает задача найти пример, когда условие правильности не выполняется. Проверим, что в рассматриваемом случае данная импликация справедлива.

Без ограничения общности положим $x_{i}=x_{1}$. Итак, пусть $\mu$ и $\nu$ изоморфны, $\nu=\alpha \mu$, $\mu \in \operatorname{Val}_{H_{1}}\left(\exists x_{1} u\right)=\exists x_{1} \operatorname{Val}_{H_{1}}(u)$. Существует такой $\mu_{1} \in \operatorname{Val}_{H_{1}}(u)$, что $\mu(y)=\mu_{1}(y)$ при всяком $y \neq x_{1}, y \in X$.

Возьмем $a=\mu_{1}\left(x_{1}\right)$. Для $\mu_{1}$ имеем последовательность $\left(a, a_{2}, \ldots, a_{n}\right)$. Напомним, что $\bar{a}=\left(a_{1}, a_{2}, \ldots, a_{n}\right)$ и $\bar{b}=\left(b_{1}, b_{2}, \ldots, b_{n}\right), b_{i}=\alpha^{\prime}\left(a_{i}\right)$, для $\mu$ и $\nu$ соответственно. Возьмем $A_{1}=\left\langle a, a_{2}, \ldots, a_{n}\right\rangle=\left\langle a,\left\langle a_{2}, \ldots, a_{n}\right\rangle\right\rangle$. Мы хотим исследовать изоморфизм $\alpha_{1}: A_{1} \rightarrow B_{1}$ с $B_{1}=\left\langle b,\left\langle b_{2}, \ldots, b_{n}\right\rangle\right\rangle$, где $b_{1}=\alpha_{1}(a), b_{i}=\alpha_{1}\left(a_{i}\right)=\alpha\left(a_{i}\right), i=2, \ldots, n$. Изоморфизм $\alpha: A \rightarrow B$ индуцирует изоморфизм $\alpha^{\prime}:\left\langle a_{2}, \ldots, a_{n}\right\rangle \rightarrow\left\langle b_{2}, \ldots, b_{n}\right\rangle$. Предположим вначале, что $a \in\left\langle a_{2}, \ldots, a_{n}\right\rangle$. В этом случае имеем изоморфизм $\alpha_{1}: A_{1} \rightarrow B_{1}$, удовлетворяющий $\alpha_{1}(a)=\alpha^{\prime}(a)=b$. Пусть $a \notin\left\langle a_{2}, \ldots, a_{n}\right\rangle$. Тогда $A_{1}$ - векторное пространство размерности на единицу большей, чем размерности пространства $\left\langle a_{2}, \ldots, a_{n}\right\rangle$. Возьмем произвольный элемент $b \in H_{2}$, не лежащий в $\left\langle b_{2}, \ldots, b_{n}\right\rangle$. Имеем векторное пространство $B_{1}=\left\langle b,\left\langle b_{2}, \ldots, b_{n}\right\rangle\right\rangle$ той же самой размерности, что и $A_{1}$. Допустив $\alpha_{1}(a)=b$, определим изоморфизм $\alpha_{1}: A_{1} \rightarrow B_{1}$, расширяющий изоморфизм $\alpha^{\prime}$.

Далее, возьмем точку $\nu_{1}: W \rightarrow H_{2}$, заданную правилом $\nu_{1}\left(x_{i}\right)=\alpha_{1} \mu_{1}\left(x_{i}\right), i=1, \ldots, n$. Здесь $\mu_{1}$ и $\nu_{1}$ изоморфны. Поскольку $\mu_{1} \in \operatorname{Val}_{H_{1}}(u)$, имеем $\nu_{1} \in \operatorname{Val}_{H_{1}}(u)$ в силу правильности формулы $u$. Точки $\nu$ и $\nu_{1}$ совпадают на переменных $x_{2}, \ldots, x_{n}$ по построению. Значит, $\nu \in$ $\operatorname{Val}_{H_{2}}\left(\exists x_{1} u\right)=\exists x_{1} \operatorname{Val}_{H_{2}}(u)$. Аналогично, если $\nu \in \exists x_{1} \operatorname{Val}_{H_{2}}(u)$, то $\mu=\alpha^{-1} \nu \in \operatorname{Val}_{H_{1}}\left(\exists x_{1} u\right)$.

Покажем, что если $u \in \Phi(Y)$ правильна, то и формула $s_{*} u \in \Phi(X)$, где $s: W(Y) \rightarrow W(X)$ и $s_{*}: \Phi(Y) \rightarrow \Phi(X)$ (определение отображения $s_{*}$ см. в параграфе 5), тоже правильна. Пусть $\mu$ и $\nu$ изоморфны, $\alpha$ - соответствующий изоморфизм и $\mu \in \operatorname{Val}_{H_{1}}\left(s_{*} u\right)=s_{*} \operatorname{Val}_{H_{1}}(u)$. Здесь $\mu s \in \operatorname{Val}_{H_{1}}(u)$. Применим изоморфизм $\alpha \mathrm{c} \nu=\alpha \mu$. Этот даст изоморфизм $(\alpha \mu) s=\nu s=\alpha(\mu s)$, и $\nu s$ и $\mu s$ изоморфны.

Предположим, что формула $u$ правильна. Тогда $\nu s \in \operatorname{Val}_{H_{2}}(u)$ и $\nu \in s_{*} \operatorname{Val}_{H_{2}}(u)=$ $\operatorname{Val}_{H_{2}}\left(s_{*} u\right)$. Формула $s_{*} u$ тоже правильна. Используя определение алгебры $\Phi(X)$ и тот факт, что все равенства правильны, можно заключить, что все $u \in \Phi(X)$ правильны. Если $\mu$ и $\nu$ изоморфны, то $\mu \in \operatorname{Val}_{H_{1}}(u)$ равносильно $\nu \in \operatorname{Val}_{H_{2}}(u)$ для всякой $u \in \Phi(X)$.

Пусть теперь $\mu$ и $\nu$ изоморфны. Поскольку $u$ правильна, $u \in \operatorname{LKer}(\mu)$ равносильно $u \in$ $\operatorname{LKer}(\nu)$. Таким образом, LKer $(\mu)=\operatorname{LKer}(\nu)$. Если размерность алгебр $H_{1}$ и $H_{2}$ бесконечна, для любой точки $\mu$ можно построить точку $\nu$, изоморфную ей. Значит, для всякой точки $\mu \in \operatorname{Hom}\left(W(X), H_{1}\right)$ найдется такая точка $\nu \in \operatorname{Hom}\left(W(X), H_{2}\right)$, что $\operatorname{LKer}(\mu)=\operatorname{LKer}(\nu)$.

Обратное также верно. Это означает, что $H_{1}$ и $H_{2}$ изотипны. Ясно, что они не обязательно изоморфны.

Метод, использованный нами в доказательстве вышеприведенного предложения, можно использовать также в случае, когда $H_{1}$ и $H_{2}$ - бесконечные абелевы группы конечной экспоненты $p$. С другой стороны, что можно сказать, когда $H_{1}$ и $H_{2}-$ свободные абелевы группы бесконечного ранга, или свободные некоммутативные группы бесконечного ранга? Используя рассмотрения, подобные сделанным в предложении 3.4 можно, в частности, изучать локально циклические группы без кручения, чтобы понять, будут ли они изотипными. Также это должно дать примеры изотипных, но не изоморфных алгебр.

ЗАмечАниЕ 3.5. Доказательство предложения 3.4 следует уже из того, что векторные пространства свободны. Мы привели подробное доказательство, имея в виду применимость к другим ситуациям. 


\section{4. Логически совершенные алгебры}

4.1. Вложения алгебр. Напомним, что на множестве (на аффинном пространстве) $\operatorname{Hom}(W(X), H)$ была определена эквивалентность $\rho$. Если $\mu$ и $\nu$ - две точки $W(X) \rightarrow H$, то $\mu \rho \nu$ равносильно $\operatorname{LKer}(\mu)=\operatorname{LKer}(\nu)$ (см. п. 1.5). Эти логические ядра вычисляются в алгебре формул $\Phi(X)$. Группа $\operatorname{Aut}(H)$ действует на множестве $\operatorname{Hom}(W(X), H)$ по правилу $\mu \rightarrow \mu \sigma$, $\sigma \in \operatorname{Aut}(H)$. Здесь каждое элементарное множество в $\operatorname{Hom}(W(X), H)$ инвариантно относительно действия группы $\operatorname{Aut}(H)$. Отсюда вытекает, что отношение $\mu \rho(\mu \sigma)$ всегда имеет место.

ОПРЕДЕЛЕНИЕ 4.1. Алгебра $H \in \Theta$ называется логически совершенной, если для всякого $X$ отношение $\mu \rho \nu$ выполнено тогда и только тогда, когда точки $\mu$ и $\nu$ сопряжены некоторым автоморфизмом данной алгебры $H$.

Это условие означает, что для всякого $X$ любой класс эквивалентности отношения $\rho$ является орбитой действия группы $\operatorname{Aut}(H)$. Поэтому для логически совершенных групп всякая орбита является элементарным множеством.

Сформулируем следующую проблему.

ПроБЛЕмА 4.2. Найти условия, при которых данную алгебру $H \in \Theta$ можно вложить в некоторую логически совершенную $H^{\prime} \in \Theta$.

Эта проблема тесно связана со следующими хорошо известными результатами в теории моделей (см. [2]).

1. Для любых конечного множества $X$, алгебры $H \in \Theta$ и точек $\mu, \nu: W(X) \rightarrow H$ условие $\operatorname{LKer}(\mu)=\operatorname{LKer}(\nu)$ равносильно следующему условию: для некоторого элементарного вложения $H \rightarrow G \in \Theta$ найдется такой автоморфизм $\sigma \in \operatorname{Aut}(G)$, что $\mu \sigma=\nu$.

2. Существует настолько большая алгебра $H \in \Theta$, что для всяких множеств $X$ и точек $\mu, \nu: W(X) \rightarrow H$ условие $\operatorname{LKer}(\mu)=\operatorname{LKer}(\nu)$ равносильно $\mu \sigma=\nu$ для некоторого $\sigma \in \operatorname{Aut}(H)$.

Обе эти теоремы являются теоремами существования и основываются на теореме компактности. Поэтому они в высокой степени неконструктивны. Мы интересуемся несколько другой задачей. Мы рассматриваем специальные многообразия $\Theta$ и задаемся вопросом, как осуществить переход от $H$ к $H^{\prime}$ посредством построений в $\Theta$ (см., например, проблему 4.2 , где $\Theta-$ это многообразие групп).

Итак, мы хотим понять, как выглядят логически совершенные алгебры. Первый вопрос здесь следующий.

Проблема 4.3. Для каких алгебр $H \in \Theta$ всякая $\operatorname{Aut}(H)$-орбита в $\operatorname{Hom}(W(X), H)$ будет элементарным множеством для любого конечного $X$ ?

Выше было отмечено, что если алгебра $H$ логически совершенна, то $\operatorname{Aut}(H)$-орбиты суть элементарные множества. На самом деле обратное утверждение также верно (предложение 4.4). Этот факт объясняет важность проблемы 4.3.

ПРЕДЛОЖЕНИЕ 4.4. Алгебра Н логически совершенна тогда и толъко тогда, когда всякая $\operatorname{Aut}(H)$-орбита в $\operatorname{Hom}(W(X), H)$ является элеметарным множеством для любого конечно$20 X$.

ДоказАтЕЛЬСтво. Фиксируем конечное $X$ и выберем произвольную точку $\mu: W(X) \rightarrow H$. Пусть $A$ будет $\operatorname{Aut}(H)$-орбитой, заданной точкой $\mu, \mu \in A$. Предположим, что множество $A$ элементарно. Тогда $A_{H}^{L L}=A$. Имеем $A_{H}^{L} \subset\{\mu\}_{H}^{L}=\operatorname{LKer}(\mu)$. Тогда $\{\mu\}_{H}^{L L}=\operatorname{LKer}(\mu)_{H}^{L} \subset$ $A_{H}^{L L}=A$. Точка $\mu$ принадлежит элементарному множеству $\{\mu\}_{H}^{L L}$ и, таким образом, вся орбита $A$ лежит в $\{\mu\}_{H}^{L L}$. Получаем равенство $\{\mu\}_{H}^{L L}=A$. Имеем также

$$
\{\mu\}_{H}^{L L L}=A_{H}^{L}=\{\mu\}_{H}^{L}=\operatorname{LKer}(\mu) .
$$


Итак, для всякой орбиты $A$ имеет место $A_{H}^{L}=\operatorname{LKer}(\mu)$, где $\mu$ - некоторая точка из $A$. Мы использовали то, что все орбиты $A$ суть элементарные множества. Различные типы соответствуют различным орбитам над $H$, и такие соответствия исчерпывают все типы, относящиеся к $H$.

Пусть теперь $\mu \rho \nu$, и пусть $A$ - какая-то $\operatorname{Aut}(H)$-орбита над $\mu$, а $B$ - какая-то $\operatorname{Aut}(H)$-орбита над $\nu$. Имеем $\operatorname{LKer}(\mu)=\operatorname{LKer}(\nu)$, т.е. $A_{H}^{L}=B_{H}^{L}$, и, таким образом, $A=B$, поскольку множества $A$ и $B$ элементарны. Итак, точки $\mu$ и $\nu$ принадлежат общей орбите. Это дает $\mu \rho_{0} \nu$. Значит, $\rho=\rho_{0}$, так что алгебра $H$ логически совершенна.

Определим понятие строго логически совершенных алгебр.

ОПРЕДЕЛЕНИЕ 4.5. Алгебру $H \in \Theta$ назовем строго логически совершенной, если для всяких множества $X$ и точек $\mu: W(X) \rightarrow H$ и $\nu: W(X) \rightarrow H$ условие $\operatorname{LKer}^{0}(\mu)=\operatorname{LKer}^{0}(\nu)$ влечет $\mu=\nu \sigma$ для некоторого $\sigma \in \operatorname{Aut}(H)$.

Это понятие действительно строже, чем понятие логической совершенности в использованном выше смысле. Можно, как это было сделано прежде, доказать, что алгебра $H$ строго логически совершенна, если для всякого конечного $X$ любая $\operatorname{Aut}(H)$-орбита в $\operatorname{Hom}(W(X), H)$ является элементарным множеством, задаваемым некоторым множеством формул $T \subset \Phi^{0}(X)$. В этом случае скажем, что любая орбита есть строго элементарное множество.

4.2. Метод HNN-расширений. В этом пункте опишем случай $\Theta=G r p$. Мы используем здесь метод HNN-расширений (см. [19]).

Пусть $a_{i}, b_{i}, i \in I$, - два множества элементов в группе $H$, и пусть $A$ и $B$ - подгруппы в $H$, порожденные всеми $a_{i}$ и $b_{i}$ соответственно. Рассмотрим систему равенств $t a_{i} t^{-1}=b_{i}$, $i \in I$. Доказано (теорема 1 из [19]), что такая система имеет решение для некоторого $t$, принадлежащего некоторой содержащей $H$ группе $G$ тогда и только тогда, когда переход $a_{i} \rightarrow b_{i}$ задает изоморфизм между подгруппами $A$ и $B$.

Эта теорема имеет многочисленные приложения. В частности, она влечет, что для любой группы без кручения $H$ найдется группа без кручения $G$, содержащая $H$ и такая, что есть только один нетривиальный класс сопряженных элементов в $G$. Это означает следующее. Пусть $G$ - такая группа и $W=W(x)$ - свободная циклическая группа. Рассмотрим аффинное пространство $\operatorname{Hom}(W, G)$ и какое-нибудь элементарное множество $A$, заданное одним "уравнением" $x \neq 1$. Как обычно, оно инвариантно относительно действия группы $\operatorname{Aut}(G)$. С другой стороны, любые два элемента $\mu$ и $\nu$ в данном элементарном множестве сопряжены некоторым элементом в $\operatorname{Aut}(G)$. Значит, $A$ - единственная нетривиальная орбита группы $\operatorname{Aut}(G)$. Мы хотели бы построить такую группу $G$, в которой нечто подобное имело бы место в аффинном пространстве $\operatorname{Hom}(W(X), G)$ для каждого конечного $X$. А именно, мы хотели бы построить по данной группе $H$ такую группу $G$, что для любого $X$ в аффинном пространстве $\operatorname{Hom}(W(X), G)$ было бы лишь конечное число $\operatorname{Aut}(G)$-орбит, причем каждая орбита была бы элементарным множеством.

Начнем с ситуации, когда $H$ - множество без операций.

Будем использовать схему из доказательства предложения 2.4. Дана эквивалентность $\tau$ на множестве $X=\left\{x_{1}, \ldots, x_{n}\right\}$, рассмотрим формулы $x_{i} \equiv x_{j}$, если $x_{i} \tau x_{j}$, и $x_{i} \not \equiv x_{j}$ иначе. Пусть $u=u_{\tau}$ - конъюнкция всех таких равенств и неравенств. Перейдем к $V=\operatorname{Val}_{H}^{X}(u)$. Точка $\mu: X \rightarrow H$ принадлежит множеству $V$, если и только если $\operatorname{Ker}(\mu)=\tau$. Если $A-$ образ точки $\mu$, то получаем биекцию $X / \tau \rightarrow A$. Если $\mu$ и $\nu$ - две точки в $V$, то $\operatorname{Ker}(\mu)=\operatorname{Ker}(\nu)=\tau$, что дает следующую коммутативную диаграмму:

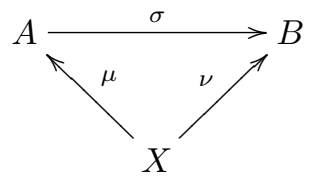


Здесь $\sigma$ - биекция, а $B$ - образ точки $\nu$. Множества $A$ и $B$ конечны, тогда как множество $H$ рассматривается как бесконечное. Имеем $A \cup A^{\prime}=H=B \cup B^{\prime}$, где $A^{\prime}$ и $B^{\prime}$ имеют одну и ту же мощность. Это ведет к расширению биекции $\sigma$ до перестановки $\sigma \in S_{H}$. Согласно диаграмме $\nu=\mu \sigma$ и $\sigma\left(a_{i}\right)=b_{i}$, где $a_{i}=\mu\left(x_{i}\right)$ и $b_{i}=\nu\left(x_{i}\right)$. Значит, всякое $V_{\tau} \tau=\operatorname{Val}_{H}^{X}\left(u_{\tau}\right)$ является орбитой группы перестановок $S_{H}$ и все орбиты таковы. Таким образом, мы получили конечное число конечно определенных орбит.

4.3. Теорема вложения. В доказательстве теоремы вложения будем использовать следующую теорему 2 из [19].

Пусть $\eta_{i}: A_{i} \rightarrow B_{i}, i \in I,-$ изоморфизмъ подгрупп группь $H$. Существует группа $G=$ $\langle H, F\rangle$, где $F$ свободно порождается элементами $t_{i}$, так что $\eta_{i}(a)=t_{i} a t_{i}{ }^{-1}$ для каждого $a \in A_{i}$.

Нашей ближайшей целью является следующая теорема.

Теорема 4.6. Каждую группу можно вложить в логически совершенную группу.

Эта теорема кажется частным случаем аналогичного теоретико-модельного результата. Мы предоставляем здесь независимое теоретико-групповое доказательство, использующее HNN-теорию.

ДокАЗАТЕЛЬСтво. Пусть $\bar{a}=\left(a_{1}, \ldots, a_{n}\right)$ и $\bar{b}=\left(b_{1}, \ldots, b_{n}\right)$ - две последовательности элементов в $H$, и пусть $A$ и $B$ - подгруппы, порожденные элементами $a_{1}, \ldots, a_{n}$ и $b_{1}, \ldots, b_{n}$ соответственно. Согласно теореме 1 из [19] существует группа $G=G_{(\bar{a}, \bar{b})}$, содержащая $H$ и такая, что некоторый автоморфизм группы $G$ переводит $\bar{a}$ в $\bar{b}$ тогда и только тогда, когда он определяет изоморфизм $\eta: A \rightarrow B$. Имея это в виду, рассмотрим эквивалентность $\tau$, определенную в $H^{(n)}$, где $n$ фиксировано. Определим $\bar{a} \tau \bar{b}$, если найдется изоморфизм $\eta=\eta_{(\bar{a}, \bar{b})}: A \rightarrow B$, расширяющий соответствие $a_{i} \rightarrow b_{i}$. Если $\bar{a} \tau \bar{b}$ верно, имеем такую группу $G=G_{(\bar{a}, \bar{b})}$ с элементом $t=t_{(\bar{a}, \bar{b})}$, что $t$ задает внутренний автоморфизм группы $G$, переводящий $\bar{a}$ в $\bar{b}$. Группу $G$ можно представить как $G=\langle H, t\rangle$. Эквивалентность $\tau$ автоматически продолжается на пространство $\operatorname{Hom}(W(X), H), X=\left\{x_{1}, \ldots, x_{n}\right\}$.

Далее, рассмотрим отношения $\tau_{n}$ для каждого натурального $n$. Отношения $\tau_{n}$ определены на всех последовательностях $\bar{a}=\left(a_{1}, \ldots, a_{n}\right)$ длины $n$. Любой такой последовательности соответствует подгруппа $A$ в $H$, порожденная элементами $a_{1}, \ldots, a_{n}$; пишем $A=A(\bar{a})$. Как и выше, $\bar{a} \tau_{n} \bar{b}$, если переходы $a_{i} \rightarrow b_{i}$ задают изоморфизм $\eta_{(\bar{a}, \bar{b})}: A(\bar{a}) \rightarrow B(\bar{b})$. Рассмотрим элементы $t_{(\bar{a}, \bar{b})}$, свободно порождающие группу $F_{n}$. Согласно [19] имеем $G_{n}=\left\langle H, F_{n}\right\rangle$, так что всякий элемент $t_{(\bar{a}, \bar{b})}$ задает некоторый внутренний автоморфизм группы $G_{n}$, который подобно $\eta_{(\bar{a}, \bar{b})}$ переводит $a_{i}$ в $b_{i}$.

На следующем шаге варьируем $n$ и рассматриваем отношения $\tau_{n}$ для разных $n$. Возьмем различные $t_{(\bar{a}, \bar{b})}$, удовлетворяющие $\bar{a} \tau_{n} \bar{b}$ для всех $n$. Рассмотрим порожденную этими $t_{(\bar{a}, \bar{b})}$ свободную группу $F$. Тогда мы имеем группу $G=\langle H, F\rangle$. Если $\bar{a} \tau_{n} \bar{b}$, то $t_{(\bar{a}, \bar{b})}$ индуцирует изоморфизм $\eta_{(\bar{a}, \bar{b})}: A(\bar{a}) \rightarrow B(\bar{b})$. Все это выполняется в силу теоремы 2 из [19].

Обозначит группу $G=\langle H, F\rangle$ через $H^{\prime}$. Итерируя переход $H \rightarrow H^{\prime}$, получим возрастающую последовательность групп $H, H^{\prime}, H^{\prime \prime}, \ldots$ Обозначим через $H^{0}$ объединение всех этих групп. Рассмотрим последовательности $\bar{a}=\left(a_{1}, \ldots, a_{n}\right)$, где $a_{i} \in H^{0}$. Для всякого $n$, если $\bar{a}\left(\tau_{n}\right) \bar{b}$, то существует такой элемент $t \in H^{0}$, что внутренний автоморфизм $\widehat{t}$ преобразует $\bar{a}$ в $\bar{b}$.

Покажем теперь, что для всякого $X=\left\{x_{1}, \ldots, x_{n}\right\}$ в $\operatorname{Hom}\left(W(X), H^{0}\right)$ равенство $\tau=\tau_{n}=\rho_{0}$ выполнено и любой класс эквивалентности $\tau$ является $\operatorname{Aut}\left(H^{0}\right)$-орбитой.

Рассмотрим $\operatorname{Hom}\left(W(X), H^{0}\right)$. Возьмем две точки $\mu, \nu: W(X) \rightarrow H^{0}$. Условие $\mu \tau \nu$ означает, что $\mu \tau_{n} \nu$ для некоторого $n$, и если $\left(\mu\left(x_{1}\right), \ldots, \mu\left(x_{n}\right)\right)=\bar{a}=\left(a_{1}, \ldots, a_{n}\right)$ и $\left(\nu\left(x_{1}\right), \ldots, \nu\left(x_{n}\right)\right)=$ $\bar{b}=\left(b_{1}, \ldots, b_{n}\right)$, то $\bar{a} \tau_{n} \bar{b}$. Мы имеем внутренний автоморфизм $\sigma=\widehat{t}$, преобразующий $\bar{a}$ в $\bar{b}$ и 
одновременно $\mu$ в $\nu$. Итак, $\mu \tau \nu$ влечет $\mu \sigma=\nu$ для некоторого $\sigma \in \operatorname{Aut}\left(H^{0}\right), \tau \subset \rho_{0}$. Kроме того, $\rho_{0} \subset \tau$, откуда $\tau=\rho_{0}$. Имеем также $\tau=\rho$. Наконец, $\rho=\rho_{0}$ и группа $H^{0}$ логически совершенна.

ЗАмечание 4.7. Bce Aut $H^{0}$-орбиты в $\operatorname{Hom}\left(W(X), H^{0}\right)$ суть элементарные множества при всяком конечном $X$. Однако число орбит бесконечно и равно числу различных ядер $\operatorname{Ker}(\alpha)$ для точек $\alpha: W(X) \rightarrow H^{0}$. Эта группа не столь хороша для обобщений примера группы с единственным нетривиальным класс сопряженности (см. [19], [20]).

С другой стороны, в предложении 2.4 мы построили группу $H$ с конечным числом Aut $H$-орбит, каждая из которых является элементарным множеством. Группа $H$ является логически совершенной группой.

Напомним, что группа $G$ называется однородной, если всякий изоморфизм $\eta: A \rightarrow B$ ее конечно порожденных подгрупп осуществляется некоторым внутренним автоморфизмом группы $G$. Такие группы были построены Холлом, Кегелем, Нейманном (см. [21]-[23]). Легко видеть, что всякая группа такого рода логически совершенна, ибо здесь имеют место равенства $\tau=\rho_{0}$ и $\rho=\rho_{0}$. Свойство однородности рассматривается в теории моделей по отношению к произвольным алгебраическим системам. В этом случае автоморфизм системы не предполагается внутренним. Понятие логически совершенной алгебры близко к понятию однородной алгебры.

Легко видеть, что алгебра $H$ однородна тогда и только тогда, когда при всяком $X$ имеет место $\tau=\rho_{0}$.

Мы сохраняем термин "совершенность", имея в виду, что в группах совершенность реализуется, как правило, посредством внутренних автоморфизмов (подобно тому, как это было в теореме 4.6).

ЗАмечАниЕ 4.8. Если в многообразии $\Theta$ все алгебры $W(X)$ конечны, а $H \in \Theta$ однородна, то $H$ автоморфно финитарна (ср. предложение 2.4).

В общем, логически совершенная алгебра не обязательно однородна: может случится, что $\rho=\rho_{0}$, но $\tau \neq \rho$. Тем не менее, справедлива следующая теорема.

Теорема 4.9 (Г.И. Житомирский). Алгебра $Н$ в $\Theta$ однородна, если и только если $Н$ строго логически совершенна.

ДоКАЗАТЕльство. Мы используем понятие правильной формулы (см. предложение 3.4). Напомним это понятие применительно к алгебре $\Phi^{0}$. Формула $u \in \Phi^{0}$ называется правильной, если для всяких изоморфных точек $\mu$ и $\nu$ включение $\mu \in \operatorname{Val}_{H_{1}}(u)$, т.е. $u \in \operatorname{LKer}^{0}(\mu)$, выполнено тогда и только тогда, когда $u \in \operatorname{LKer}^{0}(\nu)$, т.е. $\nu \in \operatorname{Val}_{H_{2}}(u)$.

Если любая формула $u \in \Phi^{0}(X)$ правильна, то $\operatorname{LKer}^{0}(\mu)=\operatorname{LKer}^{0}(\nu)$, как только $\mu$ и $\nu$ изоморфны.

Пусть теперь $H$ строго логически совершенна. Используя индукцию по мощности множества $X$, докажем, что любая формула $u \in \Phi^{0}(X)$ правильна. Пусть $X=\{x\}$. Тогда всякая булева формула над равенствами правильна. Формула $\exists x и$ имеет своими значениями 0 и 1. Поэтому для одноэлементного $X$ правильность имеет место. Пусть $X=\left\{x_{1}, \ldots, x_{n}\right\}$, и пусть для любого $X$ мощности $|X|<n$ требуемое свойство доказано. Прежде всего заметим, что свойство правильности сохраняется при булевых операциях. Пусть формула $u \in \Phi^{0}(X)$ правильна. Покажем, что $\exists x_{n} u$ правильна. Пусть даны точки $\mu, \nu: W(X) \rightarrow H$, и пусть $\mu_{0}, \nu_{0}-$ их ограничения на $X_{0}=\left\{x_{1}, \ldots, x_{n-1}\right\}$. Пусть $A, B$ - образы в $H$ точек $\mu, \nu$ соответственно, а $A_{0}, B_{0}$ - соответствующие образы их ограничений $\mu_{0}, \nu_{0}$. Пусть $\alpha$ - некоторый изоморфизм $A \rightarrow B$. Обозначим через $\alpha_{0}$ изоморфизм $A_{0} \rightarrow B_{0}$, индуцированный $\alpha$. Возьмем $\alpha \mu=\nu$ и $\alpha_{0} \mu_{0}=\nu_{0}$. Поскольку всякая формула в $\Phi^{0}\left(X_{0}\right)$ правильна, имеем $\operatorname{LKer}^{0}\left(\mu_{0}\right)=\operatorname{LKer}^{0}\left(\nu_{0}\right)$ для изоморфных $\mu_{0}, \nu_{0}$. Теперь, поскольку $H$ строго логически совершенно, имеем такой автоморфизм $\sigma \in$ Aut $H$, что $\mu_{0} \sigma=\nu_{0}$. Возьмем $\mu \sigma$ и $\nu$. Их ограничения на $X_{0}$ совпадают. 
Пусть теперь $\mu \in \operatorname{Val}_{H}^{X}\left(\exists x_{n} u\right)=\exists x_{n} \operatorname{Val}_{H}^{X}(u)$. Тогда найдется такая точка $\mu^{\prime} \in \operatorname{Val}_{H}^{X}(u)$, что $\mu$ и $\mu^{\prime}$ совпадают на $X_{0}$. Тогда $\mu \sigma$ и $\mu^{\prime} \sigma$ совпадают на $X_{0}$. Однако $\mu \sigma$ и $\nu$ совпадают на $X_{0}$. Тогда $\mu^{\prime} \sigma=\nu^{\prime}$ и $\nu$ совпадают на $X_{0}$. Кроме того, наряду с $\mu^{\prime}$ точка $\mu^{\prime} \sigma=\nu^{\prime}$ принадлежит множеству $\operatorname{Val}_{H}^{X}(u)$. Поэтому $\nu \in \operatorname{Val}_{H}^{X}\left(\exists x_{n} u\right)$. Значит, $\mu \in \operatorname{Val}_{H}^{X}\left(\exists x_{n} u\right)$ влечет $\nu \in \operatorname{Val}_{H}^{X}\left(\exists x_{n} u\right)$. Подобным же образом можно убедиться, что $\nu \in \operatorname{Val}_{H}^{X}\left(\exists x_{n} u\right)$ влечет $\mu \in \operatorname{Val}_{H}^{X}\left(\exists x_{n} u\right)$. Таким образом, формула $\exists x_{n} u$ также правильна. Следовательно, все формулы из $\Phi^{0}(X)$ правильны. Итак, если $\mu$ и $\nu$ изоморфны, то $\operatorname{LKer}^{0}(\mu)=\operatorname{LKer}^{0}(\nu)$. Воспользовавшись еще раз условием строгой логической совершенности, получим $\mu \sigma=\nu$ для некоторого $\sigma \in$ Aut $H$, расширяющего $\alpha: A \rightarrow B$. Следовательно, алгебра $A$ однородна.

Докажем импликацию в другую сторону. Пусть $H$ однородна. Тогда $\mu \tau \nu$ означает, что $\mu \sigma=\nu$ для некоторого $\sigma \in$ Aut $H$. Поэтому класс эквивалентности $\tau$ будет некоторой орбитой. Однако, каждый класс эквивалентности $\tau$ задается каким-то множеством $T \subset \Phi^{0}(X)$. Следовательно, алгебра $H$ строго логически совершенна.

ЗАмечание 4.10. Теорему 4.9 можно вывести из критерия $\tau=\rho_{0}$. Тем не менее, мы представили здесь исходное доказательство.

ЗАмЕчАниЕ 4.11. Из доказательства теоремы 4.6 можно усмотреть, что всякая группа вкладывается в однородную группу. Этот факт можно также вывести из метода HNN-расширений.

Теперь приведем пример логически совершенной, но не однородной группы. Этот пример также принадлежит Житомирскому.

\section{ПреДлОЖЕнИЕ 4.12. Бесконечная ииклическая группа $\mathbb{Z}$ логически совершенна.}

ДокАзАтЕЛЬСтво. Рассмотрим бесконечную циклическую группу, представленную как аддитивная группа целых чисел $\mathbb{Z}$. Эта группа является также $\mathbb{Z}$-модулем. Каждая подгруппа группы $\mathbb{Z}$ является ее подмодулем. Возьмем в качестве $\Theta$ многообразие всех абелевых групп либо многообразие всех $\mathbb{Z}$-модулей. Ясно, что группа $\mathbb{Z}$ не однородна. Проверим, что $\mathbb{Z}$ логически совершенна.

Возьмем какое-нибудь множество $X=\left\{x_{1}, \ldots, x_{n}\right\}$. Выберем переменную $y$ и положим $Y=\{y, X\}$. Зададим отображение $s: Y \rightarrow X$ правилом $s\left(x_{i}\right)=x_{i}, s(y)=0$. Таким образом, имеем морфизмы $s: W(Y) \rightarrow W(X)$ и $s_{*}: \Phi(Y) \rightarrow \Phi(X)$. Для каждой формулы $v \in \Phi(Y)$ найдется формула $u=s_{*} v, u \in \Phi(X)$, не обязательна принадлежащая $\Phi^{0}(X)$.

Пусть $\mu: W(X) \rightarrow \mathbb{Z}$ - некоторая точка. Имеем $\mu \in \operatorname{Val}_{\mathbb{Z}}^{X}(u)=s_{*} \operatorname{Val}_{\mathbb{Z}}^{Y}(v)$, если и только если $\mu s \in \operatorname{Val}_{\mathbb{Z}}^{Y}(v)$.

Возьмем две точки $\mu, \nu: W(X) \rightarrow \mathbb{Z}$, и пусть $\operatorname{LKer}(\mu)=\operatorname{LKer}(\nu)$. Покажем, что $\mu$ и $\nu$ сопряжены посредством некоторого автоморфизма группы $\mathbb{Z}$. Эта группа помимо тождественного автоморфизма имеет только автоморфизм, переводящий данный элемент в обратный ему. Проверим, что либо $\mu=\nu$, либо $\mu=-\nu$.

Пусть $\mu\left(x_{i}\right)=a_{i}, \nu\left(x_{i}\right)=b_{i}, i=1, \ldots, n$. Если все $a_{i}$ равны нулю, то $\mu=\nu$. Поэтому можно предполагать, что $a_{1} \neq 0$.

Для точки $\mu$ мы намереваемся построить специальную проверяющую формулу $v \in \Phi(Y)$, удовлетворяющую $\mu \in \operatorname{Val}_{\mathbb{Z}}^{X}(u), u=s_{*} v$. Отсюда будет следовать $\nu \in \operatorname{Val}_{\mathbb{Z}}^{X}(u)$. Вначале определим $v_{0} \in \Phi(Y)$. Затем $v$ строится как $v=\exists y v_{0}$.

Пусть $v_{0}$ - формула

$$
\left(x_{1} \equiv\left|a_{1}\right| y\right) \wedge\left(x_{2} \equiv \operatorname{sgn}\left(a_{2} a_{1}\right)\left|a_{2}\right| y\right) \wedge \cdots \wedge\left(x_{n} \equiv \operatorname{sgn}\left(a_{n} a_{1}\right)\left|a_{n}\right| y\right) .
$$

Здесь $|a|$ обозначает абсолютное значение $a$, тогда как $\operatorname{sgn}(a)$ есть знак $a$.

Зададим точку $\gamma: W(Y) \rightarrow \mathbb{Z}$ следующим образом: $\gamma\left(x_{i}\right)=\mu\left(x_{i}\right)=a_{i}$ и $\gamma(y)=\operatorname{sgn}\left(a_{1}\right) 1$. Точка $\gamma$ удовлетворяет $v_{0}$. Поскольку $\mu s$ и $\gamma$ совпадают на $X$, точка $\mu s$ удовлетворяет формуле 
$\exists y v_{0} \equiv v$. Другими словами, $\mu \in \operatorname{Val}_{\mathbb{Z}}^{X}(u)$, где $u=s_{*} v$. Имеем также $\nu s \in \operatorname{Val}_{\mathbb{Z}}^{Y}(v)$. Отсюда вытекает, что для некоторых значений $c$ переменной $y$ и значений $b_{i}$ переменных $x_{i}$ верно

$$
b_{1}=\left|a_{1}\right| c, \quad \ldots, \quad b_{n}=\operatorname{sgn}\left(a_{n} a_{1}\right)\left|a_{n}\right| c .
$$

Это показывает, что $b_{i}=a_{i} c$, если $a_{1}>0$, и $b_{i}=-a_{i} c$, если $a_{1}<0$. Меняя местами $\mu$ и $\nu$, получаем такое $d$, что $a_{i}=b_{i} d$, если $b_{1}>0$, и $a_{i}=-b_{i} d$, если $b_{1}<0$. Отсюда приходим к равенству $c=1$ или $c=-1$. В первом случае имеем $\mu=\nu$, а во втором $-\mu=-\nu$. Доказательство предложения закончено.

Группа $\mathbb{Z}$ не является строго логически совершенной, так как $\mathbb{Z}$ не однородна. Было бы интересно привести другие примеры, работая с другими многообразиями $\Theta$.

ЗАмЕчАниЕ 4.13. Соображения, аналогичные используемым в предложении 4.12, позволяют доказать, что свободная абелева группа конечного ранга логически совершенна.

Сделаем еще одно замечание. Возьмем $\mu: W(X) \rightarrow H$ и рассмотрим его замыкание $A=\{\mu\}_{H}^{L L}=(\operatorname{LKer}(\mu))_{H}^{L}$. Это $A$ является минимальным элементарным множеством, а всякое элементарное множество в $\operatorname{Hom}(W(X), H)$ является объединением таких попарно непересекающихся $A$. Если $H$ логически совершенна, то эти минимальные множества совпадают с орбитами группы $\operatorname{Aut}(H)$ (это свойство решетки элементарных множеств в $\operatorname{Hom}(W(X), H))$. Понятно, что минимальные элементарные множества находятся во взаимно однозначном соответствии с типами.

Теперь приведем два примера строго логически совершенных абелевых групп.

4.4. Пример. Исследуем один конкретный пример. Пусть группа $H$ является дискретным прямым произведением все простых циклических групп различных простых порядков. Покажем, что для данной $H$ все $\operatorname{Aut}(H)$-орбиты в $\operatorname{Hom}(W, H)$ будут элементарными множествами.

Исходя из переменной $x \in W=W(X)$ выпишем формулу $u=u(x)$ вида $x \neq 1 \wedge x^{m}=1 \wedge u_{0}$, где $u_{0}$ - конъюнкция всех $x^{m_{i}} \neq 1$ по всем делителям $m_{i}$ числа $m$.

Только элементы $g$ порядка $m$ удовлетворяют формуле $u=u(x)$. Есть лишь конечное число таких элементов, а именно $\left(p_{1}-1\right), \ldots,\left(p_{k}-1\right)$, если $m=p_{1} \cdots p_{k}$.

Возьмем конечное $X$ и рассмотрим $\operatorname{Hom}(W(X), H)$. Пусть $\left(\mu\left(x_{1}\right), \ldots, \mu\left(x_{n}\right)\right)=\bar{g}=$ $\left(g^{1}, \ldots, g^{n}\right)$ для некоторой точки $\mu: W(X) \rightarrow H$. Представим каждый элемент $g^{i}$ как $g^{i}=$ $g_{1}^{i} \cdots g_{k_{i}}^{i}$. Множители упорядочены по возрастанию их простых порядков. Порядок элемента $g^{i}$ есть некоторое число $m^{i}$.

Далее работаем с множеством формул $u\left(x_{1}\right), \ldots, u\left(x_{n}\right)$ порядков $m_{1}, \ldots, m_{n}$; пусть $u-$ их конъюнкция. Перейдем к элементарному множеству $\operatorname{Val}_{H}^{X}(u)=A$. Bсе точки $\mu: W(X) \rightarrow H$ вышеописанного вида включаются в данное множество.

ПрЕДЛОЖЕНИЕ 4.14. Всякое $A=\operatorname{Val}_{H}^{X}(u)$ является орбитой группы Aut $(H)$ и всякая ее орбита имеют такой вид.

Доказательство. Пусть $\mu: W(X) \rightarrow H$ - какая-нибудь точка в $A$. Для всякого автоморфизма $\sigma \in \operatorname{Aut}(H)$ выполнено включение $\mu \sigma \in A$. Нужно проверить, верно ли $\nu=\mu \sigma$ для любой точки $\nu: W(X) \rightarrow H$ из $A$ и некоторого автоморфизма $\sigma$.

По определению имеем $\mu \sigma(x)=\mu(x) \circ \sigma=g \circ \sigma$ для всякого $x \in X$. Пусть $g=g_{1} \cdots g_{k}$, где порядок элемента $g_{i}$ есть $p_{i}$. Пусть, кроме того, $\nu(x)=g^{\prime}=g_{1}^{\prime} \cdots g_{k}^{\prime}$, где порядок элемента $g_{i}^{\prime}$ также есть $p_{i}$, и элементы $g_{i}$ и $g_{i}^{\prime}$ является образующими циклической группы порядка $p_{i}$. Следовательно, переход $g_{i} \rightarrow g_{i}^{\prime}$ задает автоморфизм этих циклических подгрупп. Это верно для каждого $x \in X$, значит, мы получаем автоморфизм $\sigma$, переводящий $g$ в $g^{\prime}$. Имеем также $\mu \sigma=\nu$. 
Докажем теперь, что всякая орбита является некоторым множеством $A$ такого вида. Возьмем произвольные $X=\left\{x_{1}, \ldots, x_{n}\right\}$ и точку $\mu: W(X) \rightarrow H$. Этой точке соответствует последовательность $\bar{g}=\left(g^{1}, \ldots, g^{n}\right)$. Множество $A$, содержащее точку $\mu$, соответствует самой $\mu$, и это верно для всякого $A$. Применяя к $\bar{g}$ произвольные автоморфизмы $\sigma \in \operatorname{Aut}(H)$, получим всю орбиту $A$.

Существует бесконечно много различных орбит и, значит, бесконечно много элементарных множеств и типов. Интересно то, что всякая орбита конечно определена. Легко понять, что группа $H$ не удовлетворяет никакому из условий нётеровости; значит, если некая группа $H^{\prime}$ изотипна с $H$, то $H$ и $H^{\prime}$ изоморфны. На самом деле, для этого результата достаточно уже элементарной эквивалентности $H$ и $H^{\prime}$. Докажем этот факт и сделаем затем несколько замечаний, касающихся нётеровости.

Пусть $H$ - группа из предложения 4.14, а $H^{\prime}$ - группа, изотипная с $H$. Тогда они элементарно эквивалентны и, значит, удовлетворяют одним и тем же тождествам. Поэтому $H^{\prime}$ абелева. Формула $\exists x\left(x^{p} \equiv 1\right)$ в $H$ показывает, что также и в $H^{\prime}$ существуют элементы порядка $p$. Формула

$$
x_{1}^{p} \equiv x_{2}^{p} \equiv 1 \rightarrow x_{1} \equiv x_{2}^{m_{1}} \vee \cdots \vee x_{1} \equiv x_{2}^{m_{k}},
$$

где $m_{i}<p$, влечет, что $H^{\prime}$ содержит лишь одну циклическую подгруппу порядка $p$. Формула $x^{p^{n}} \equiv 1 \rightarrow x^{p} \equiv 1$ означает, что все $p$-элементы имеют порядок $p$.

Все это верно в $H^{\prime}$. Следовательно, $H^{\prime}$ является прямым произведением всех циклических подгрупп всех простых порядков, что дает искомый изоморфизм.

Покажем, что $H$ не является логически нётеровой. Возьмем бесконечное подмножество $M$ множества простых, имеющее бесконечное дополнение $M^{\prime}$. Рассмотрим формулу $\neg\left(x^{p} \equiv 1 \rightarrow\right.$ $x \equiv 1)$ по всем $p \in M$. Это множество не сводится к конечному, ибо $M^{\prime}$ бесконечно.

4.5. Аддитивная группа рациональных чисел. Будем работать теперь с аддитивной группой $H$ рациональных чисел.

Прежде всего покажем, что эта группа однородна. Фиксируем множество $X=\left\{x_{1}, \ldots, x_{n}\right\}$. Пусть $W(X)$ - свободная абелева группа над $X$. Возьмем точки $\mu, \nu: W(X) \rightarrow H$. Пусть $A$ и $B$ - образы точек $\mu \nu$ соответственно, и пусть $\alpha: A \rightarrow B$ - некоторый изоморфизм. Группы $A$ и $B$ циклические и, таким образом, $A=\{a\}, B=\{b\}$. Предположим, что $\alpha(a)=b$. Пусть последовательность $a_{1}, \ldots, a_{n}$, где $a_{i}=m_{i} a \in A$, соответствует точке $\mu$. Поэтому имеем $\left(b_{1}, \ldots, b_{n}\right)$ для $\nu$. Здесь $\alpha\left(a_{i}\right)=b_{i}=m_{i} b$, где все $m_{i}$ целые. Возьмем $s=b / a, s a=b$. Умножение элемента $h \in H$ на $s$ является автоморфизмом группы $H$. Этот автоморфизм продолжает исходный изоморфизм $\alpha$.

Далее, если $A$ - орбита точки $\mu$, то эта орбита определяется множеством $T$ формул, заданных ядром точки $\mu$.

Ту же самую орбиту можно описать, используя иной подход. Пусть $x$ - некоторая вспомогательная переменная; рассмотрим формулу

$$
u=u\left(m_{1}, \ldots, m_{n}\right)=\exists x\left(x^{m_{1}}=x_{1} \wedge \ldots \wedge x^{m_{n}}=x_{n}\right) .
$$

Если ограничиться значением этой формулы на исходном множестве $X$, получится искомая орбита.

Далее в определении 4.15 и теореме 4.16 возможное использование вспомогательной переменной $x$ принято во внимание.

ОПРЕДЕЛЕНИЕ 4.15. Алгебра $H$ называется логически локалъно нётеровой, если для каждого конечного множества $X$ замыкание конечного множества точек $\mu_{i}: W(X) \rightarrow H$ конечно определено.

Теорема 4.16. Локально ииклическал группа $H$ логически локально нётерова. 
ДоказАтельство. Прежде всего покажем, что для всякой точки $\mu: W(X) \rightarrow H$ имеет место $\operatorname{LKer}(\mu)=\{u\}_{H}^{L L}$ с некоторой формулой $u$ вида $u=u\left(m_{1}, \ldots, m_{n}\right)$. Кроме того, $\{\mu\}_{H}^{L L}$ является конечно определимым замыканием точки $\mu$.

Пусть $u=u\left(m_{1}, \ldots, m_{n}\right)$. Тогда $\operatorname{Val}_{H}^{X}(u)=\{u\}_{H}^{L}$ и $\left(\operatorname{Val}_{H}^{X}(u)\right)_{H}^{L}=\{u\}_{H}^{L L}$. Пусть теперь $\mu \in$ $A_{0}=\operatorname{Val}_{H}^{X}(u)$. Тогда $\{\mu\}_{H}^{L} \supset A_{0 H}^{L}=\{u\}_{H}^{L L}$ и $\operatorname{LKer}(\mu) \supset\{u\}_{H}^{L L}$. По определению оператора $L$ имеем $A_{0}^{L}=\bigcap_{\nu \in A_{0}} \operatorname{LKer}(\nu)$. Из предыдущих рассмотрений следует, что $\mu$ и $\nu$ изотипны. Значит, $A_{0 H}^{L}=\operatorname{LKer}(\mu)=\{u\}_{H}^{L L}$. Имеем также $(\operatorname{LKer}(\mu))_{H}^{L}=A_{0}$ для произвольной точки $\mu \in A_{0}$.

Нам нужно еще одно замечание, касающееся решетки всех элементарных множеств в данном $\operatorname{Hom}(W(X), H)$. Пусть $A$ и $B$ - два такие множества, $A_{H}^{L}=T_{1}, B_{H}^{L}=T_{2}$. Пусть $T_{1}^{0}-$ некоторое подмножество множества $T_{1}$, удовлетворяющее $\left(T_{1}^{0}\right)_{H}^{L L}=T_{1}$. Возьмем подмножество $T_{2}^{0}$ множества $T_{2}$, удовлетворяющее $\left(T_{2}^{0}\right)_{H}^{L L}=T_{2}$. Обозначим через $T_{1}^{0} \vee T_{0}^{2}$ множество всех $u \vee v, u \in T_{1}^{0}, v \in T_{2}^{0}$. Проверим, что $\left(T_{1}^{0} \vee T_{0}^{2}\right)_{H}^{L}=A \cup B$. В самом деле,

$$
\begin{aligned}
\left(T_{1}^{0} \vee T_{0}^{2}\right)_{H}^{L}=\bigcap_{u \vee v} \operatorname{Val}_{H}^{X}(u \vee v) & =\bigcap_{u \vee v}\left(\operatorname{Val}_{H}^{X}(u) \cup \operatorname{Val}_{H}^{X}(v)\right) \\
& =\left(\bigcap_{u \in T_{1}^{0}} \operatorname{Val}_{H}^{X}(u)\right) \cup\left(\bigcap_{v \in T_{2}^{0}} \operatorname{Val}_{H}^{X}(v)\right)=A \cup B .
\end{aligned}
$$

Применим это к группе $H$. Пусть $u_{1}=u\left(m_{1}, \ldots, m_{n}\right), u_{2}=u\left(m_{1}^{\prime}, \ldots, m_{n}^{\prime}\right), A=\operatorname{Val}_{H}^{X}\left(u_{1}\right)$, $B=\operatorname{Val}_{H}^{X}\left(u_{2}\right)$. Тогда $A \cup B=\operatorname{Val}_{H}^{X}\left(u_{1} \vee u_{2}\right)$. Это показывает, что объединение в любом конечном числе множеств вида $A$ является элементарным конечно определенным множеством.

Далее, пусть $A_{0}$ - конечное множество точек $\mu: W(X) \rightarrow H$. Всякая точка $\mu \in A_{0}$ принадлежит некоторому $\operatorname{Val}_{H}^{X}(u), u=u\left(m_{1}, \ldots, m_{n}\right)$. Значит, $A_{0}$ содержится в конечном объединении множеств вида $\operatorname{Val}_{H}^{X}(u)$.

Пусть $A_{0}$ - подмножество некоторого $A=(\operatorname{LKer}(\mu))_{H}^{L}$. Тогда $A_{0}^{L}{ }_{H}=\bigcap_{\nu \in A_{0}} \operatorname{LKer}(\nu)=$ $\operatorname{LKer}(\mu)$ для некоторой точки $\mu \in A_{0}$. Замыканием будет $A_{0}^{L L}{ }_{H}=A$.

Возьмем теперь конечное множество точек $A_{0}=\left\{\mu_{1}, \ldots, \mu_{k}\right\}, \mu_{i} \in \operatorname{LKer}\left(\mu_{i}\right)_{H}^{L}$, и покажем, что его замыкание конечно определено. Имеем $A_{0 H}^{L}=\bigcap_{\mu_{i} \in A_{0}} \operatorname{LKer}\left(\mu_{i}\right)$.

Из сделанных замечаний об элементарных множествах вытекает, что

$$
A_{0}^{L L}{ }_{H}=\bigcup_{\mu_{i}} \operatorname{LKer}\left(\mu_{i}\right)_{H}^{L}=\bigcup_{\mu_{i}} A_{i}, \quad A_{i}=\operatorname{LKer}\left(\mu_{i}\right)_{H}^{L} .
$$

Для группы $H$ рациональных чисел все $A_{i}$ конечно определены. Поэтому $\bigcup_{\mu_{i}} A_{i}$ тоже конечно определено. Следовательно, замыкание $A_{0}^{L L}{ }_{H}$ конечно определено.

Теперь можно сравнить логически локальную нётеровость с другими условиями нётеровости. Мы не будем изучать этот вопрос здесь. Заметим лишь, что всякая логически нётерова алгебра $H$ является логически локально нётеровой. Однако, то, что всякая конечно порожденная подгруппа группы $H$ логически нётерова, еще не означает, что $H$ логически локально нётерова.

\section{5. Некоторые факты из алгебраической логики. Appendix}

5.1. Введение. Для всякого многообразия алгебр $\Theta$ мы различаем обычную чистую логику в $\Theta$ и алгебраическую логику в $\Theta$. Формулы алгебраической логики суть формулы чистой логики, сжатые по семантическому отношения в данном $\Theta$. Полиадические алгебры Халмоша и цилиндрические алгебры Тарского суть основные общие структуры алгебраической логики. Существенным характеристическим свойством этих структур является то, 
что они допускают бесконечное множество переменных. Обозначим это множество через $X^{0}$ (см. [11], [24]).

В нашем случае мы вынуждены рассматривать систему всех конечных подмножеств $X$ множества $X^{0}$ вместо столь большого $X^{0}$. Обозначим эту систему через $Г$. Тогда мы переходим к многосортной алгебре с системой сортов Г. Мы приходим, в частности, к многосортным алгебрам Халмоша и к категориям Халмоша.

5.2. Категория - алгебра $\operatorname{Hal}_{\Theta}(\boldsymbol{H})$. Начнем с одного важного примера, а именно с категории Халмоша $\mathrm{Hal}_{\Theta}(H)$. Здесь $H$ - некоторая алгебра в $\Theta$. Объектами этой категории служат расширенные булевы алгебры $\operatorname{Bool}(W(X), H)$. Определим морфизмы $s_{*}: \operatorname{Bool}(W(X), H) \rightarrow \operatorname{Bool}(W(Y), H)$. Обозначим через $\Theta^{0}$ категорию свободных в $\Theta$ алгебр $W=W(X), X \in \Gamma$. Имеем также категорию $K_{\Theta}^{0}(H)$ всех аффинных пространств над $H$. Ее морфизмами служат отображения $\widetilde{s}: \operatorname{Hom}(W(Y), H) \rightarrow \operatorname{Hom}(W(X), H)$, где $s: W(X) \rightarrow W(Y)$ - морфизм в $\Theta^{0}$ и $\widetilde{s}(\nu)=\nu s: W(Y) \rightarrow H$ для $\nu: W(X) \rightarrow H$. Для каждого $A \subset \operatorname{Hom}(W(X), H)$ положим $s_{*} A=\widetilde{s}^{-1} A$. Это описывает морфизм в $\operatorname{Hal}_{\Theta}(H)$. Всякий $s_{*}$ является также гомоморфизмом булевой алгебры и согласован с кванторами и равенствами (см. п. 5.4).

Переходя к общим определениям, уточним понятие расширенных булевых алгебр.

Напомним, что в алгебраической логике $(\mathrm{AL})$ кванторы трактуются как операции на булевых алгебрах. Пусть $B$ - булева алгебра. Ее экзистенциальный квантор (или квантор существования) - это отображение $\exists: B \rightarrow B$, удовлетворяющее следующим условиям:

1) $\exists 0=0$

2) $\exists a>a$;

3) $\exists(a \wedge \exists b)=\exists a \wedge \exists b$;

4) пусть $s: W(X) \rightarrow W(Y), s^{\prime}: W(Y) \rightarrow W(Z)$, и пусть $s \in \Phi(X)$; тогда $s_{*}^{\prime}\left(s_{*}(u)\right)=$ $\left(s^{\prime} s\right)_{*}(u)$.

Унивесальный квантор (или квантор всеобщности) определяется двойственно:

1) $\forall 1=1$;

2) $\forall a<a$;

3) $\forall(a \vee \forall b)=\forall a \vee \forall b$.

Здесь 0 и 1 суть нуль и единица алгебры $B$, а $a, b-$ произвольные элементы $B$. Кванторы $\exists$ и $\forall$ согласованы обычным образом: $\overline{\exists a}=\forall \bar{a}, \overline{\forall a}=\exists \bar{a}$.

Пусть $\Theta$ и $W=W(X) \in \Theta$ фиксированы и $B$ - булева алгебра. Назовем $B$ расширенной булевой алгеброй в $\Theta$ над $W(X)$, если выполнены следующие условия:

1) существуют определенные выше кванторы $\exists x$ для всех $x \in X$ в $B$, удовлетворяющие дополнительно условию $\exists x \exists y=\exists y \exists x$ для всех $x, y \in X$;

2) любой формуле $w \equiv w^{\prime}, w, w^{\prime} \in W$ соответствует константа в $B$, обозначаемая тоже через $w \equiv w^{\prime}$; здесь:

2.1) $w \equiv w$ является единицей алгебры $B$;

2.2 для всякой $n$-местной операции $\omega \in \Omega$, где $\Omega$ - сигнатура многообразия $\Theta$, выполнено

$$
w_{1} \equiv w_{1}^{\prime} \wedge \cdots \wedge w_{n} \equiv w_{n}^{\prime}<w_{1} \ldots w_{n} \omega \equiv w_{1}^{\prime} \ldots w_{n}^{\prime} \omega .
$$

Можно рассматривать многообразие таких алгебр при любых заданных $\Theta$ и $W=W(X)$.

\section{3. Категории Халмоша. Общее определение.}

ОпредЕлениЕ 5.1. Категория $\Upsilon$ называется категорией Халмоша, если выполнены следующие условия:

1) все ее объекты имеют вид $\Upsilon(X)$ и являются расширенными булевыми алгебрами в $\Theta$ над $W(X)$;

2) морфизмы имеют вид $s_{*}: \Upsilon(X) \rightarrow \Upsilon(Y)$, где $s: W(X) \rightarrow W(Y)-$ морфизмы в $\Theta^{0}, s_{*}-$ гомоморфизмы булевых алгебр, а переход $s \rightarrow s_{*}$ задается ковариантным функтором $\Theta^{0} \rightarrow \Upsilon$ 
3) имеют место тождества, контролирующие взаимодейтсвие морфизмов с кванторами и равенствами; согласованность с кванторами следующая:

3.1) $s_{1 *} \exists x a=s_{2 *} \exists x a, a \in \Upsilon(X)$, если $s_{1} y=s_{2} y$ для любого $y \in X, y \neq x$;

3.2) $s_{*} \exists x a=\exists(s x)\left(s_{*} a\right)$, если $s x=y \in Y$ и $y=s x$ не лежит в носителе $s x^{\prime}, x^{\prime} \in X$, $x^{\prime} \neq x$

4) следующие условия описывают согласованность с равенствами:

4.1) $s_{*}\left(w \equiv w^{\prime}\right)=\left(s w \equiv s w^{\prime}\right)$ для $s: W(X) \rightarrow W(Y), w, w^{\prime} \in W(X)$;

4.2) $s_{w}^{x} a \wedge\left(w \equiv w^{\prime}\right)<s_{w^{\prime}}^{x} a$ для произвольного $a \in \Upsilon(X), x \in X$, где $w, w^{\prime} \in W(X)$, a $s_{w}^{x}: W(X) \rightarrow W(X)$ задано правилом $s_{w}^{x}(x)=w, s_{w}^{x}(y)=y, y \in X, y \neq x$.

Категория $\mathrm{Hal}_{\Theta}(H)$ служит примером категории Халмоша. Другой важный пример - категория формул $\mathrm{Hal}_{\Theta}^{0}$ алгебры формул $\mathrm{Hal}_{\Theta}^{0}(X)=\Phi(X)$. Эта категория играет в логической геометрии ту же роль, что категория $\Theta^{0}$ играет в AG.

5.4. Алгебры Халмоша. Мы работаем с многосортными алгебрами Халмоша, ассоциированными с категориями Халмоша. Опишем вначале сигнатуру $L_{X}$. Для всякого $X$ положим $L_{X}=\left\{\vee, \wedge,{ }^{-}, \exists x, x \in X, M_{X}\right\}$. Здесь $M_{X}-$ множество всех равенств над алгеброй $W=W(X)$. Добавим все $s: W(X) \rightarrow W(Y)$ ко всем $L_{X}$, трактуя их как символы унарных операций. Обозначим эту новую сигнатуру через $L_{\Theta}$.

Далее, рассмотрим алгебры $\Upsilon=\left(\Upsilon_{X}, X \in \Gamma\right)$. Всякая $\Upsilon_{X}$ - это алгебра в сигнатуре $L_{X}$, причем всякому символу $s: W(X) \rightarrow W(Y)$ соответствует унарная операция (отображение) $s_{*}: \Upsilon_{X} \rightarrow \Upsilon_{Y}$

ОПРЕДЕЛЕНиЕ 5.2. Назовем алгебру $\Upsilon$ в сигнатуре $L_{\Theta}$ алгеброй Халмоша, если выполнены следующие условия:

1) всякая $\Upsilon_{X}$ является расширенной булевой алгеброй в сигнатуре $L_{X}$;

2) всякое отображение $s_{*}: \Upsilon_{X} \rightarrow \Upsilon_{Y}$ является гомоморфизмом булевых алгебр;

3) тождества, контролирующие взаимодействие операций $s_{*}$ с кванторами и равенствами, те же, что в определении категорий Халмоша.

Теперь ясно, что каждую категорию Халмоша $\Upsilon$ можно рассматривать как алгебру Халмоша и наоборот. В частности, это относится к $\mathrm{Hal}_{\Theta}(H)$. Напомним также, что гомоморфизмы многосортных алгебр действуют покомпонентно.

5.5. Категории и алгебры формул. Обозначим через $M=\left(M_{X}, X \in \Gamma\right)$ многосортное множество с компонентами $M_{X}$.

Возьмем абсолютно свободную алгебру $\Upsilon^{0}=\left(\Upsilon_{X}^{0}, X \in \Gamma\right)$ над $M$ в сигнатуре $L_{\Theta}$. Элементы каждой $\Upsilon_{X}^{0}$ суть формулы логики первого порядка (FOL), которые индуктивно построены из равенств, с использованием сигнатуры $L_{\Theta}$. Итак, $\Upsilon^{0}-$ многосортная алгебра чистых FOL формул над равенствами.

Обозначим через $\mathrm{Hal}_{\Theta}$ многообразие $\Gamma$-сортных алгебр Халмоша в сигнатуре $L_{\Theta}$. Обозначим через $\mathrm{Hal}_{\Theta}^{0}$ свободную алгебру этого мнообразия над многосортным множеством равенств $M=\left(M_{X}, X \in \Gamma\right)$.

То же самое $M$ задает гомоморфизм $\pi=\left(\pi_{X}, X \in \Gamma\right): \Upsilon^{0} \rightarrow \mathrm{Hal}_{\Theta}^{0}$. Если $u \in \Upsilon_{X}^{0}$, то образ $u^{\pi_{X}}=\bar{u}$ в $\operatorname{Hal}_{\Theta}^{0}(X)$ рассматривается как сжатая формула.

Полагая $\operatorname{Hal}_{\Theta}^{0}(X)=\Phi(X)=\left(\Upsilon_{X}^{0}\right)^{\pi_{X}}$, получаем искомую алгебру сжатых формул, (систематически) используемую нами во всей статье. Это расширенная булева алгебра с дополнительными операцями вида $s_{*}$.

Напомним, что алгебра Халмоша формул $\mathrm{Hal}_{\Theta}^{0}$ является также категорией Халмоша. Имеем ковариантный функтор $\Theta^{0} \rightarrow \mathrm{Hal}_{\Theta}^{0}$. 
5.6. Значение формулы. Значение $\operatorname{Val}_{H}^{X}\left(w \equiv w^{\prime}\right)$ соответствует каждому равенству $w \equiv w^{\prime}, w, w^{\prime} \in W(X)$. Это задает отображение $\operatorname{Val}_{H}: M \rightarrow \operatorname{Hal}_{\Theta}(H)$, расширяемое по индукции единственным образом с атомных формул до гомоморфизмов

$$
\operatorname{Val}_{H}^{0}: \Upsilon^{0} \rightarrow \operatorname{Hal}_{\Theta}(H), \quad \operatorname{Val}_{H}: \operatorname{Hal}_{\Theta}^{0} \rightarrow \operatorname{Hal}_{\Theta}(H) .
$$

Для всякого $X \in \Gamma$ имеет место следующая коммутативная диаграмма:

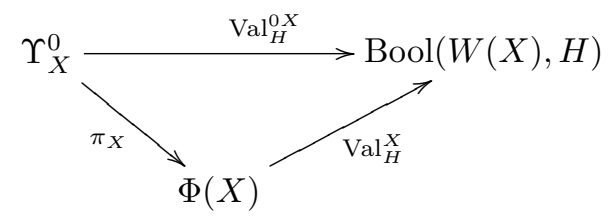

Таким образом, для всякой формулы $u \in \Upsilon_{X}^{0}$ и соответствующего $\bar{u} \in \Phi(X)$ имеются значения $\operatorname{Val}_{H}^{0 X}(u)=\operatorname{Val}_{H}^{X}(\bar{u})$.

Сделаем одно замечание о ядре гомоморфизма $\mathrm{Val}_{H}$. Имеет место

$$
\operatorname{Ker}\left(\operatorname{Val}_{H}\right)=\operatorname{Th}(H)=\left(\operatorname{Th}^{X}(H), X \in \Gamma\right) .
$$

Здесь $T h(H)=\left(\operatorname{Th}^{X}(H), X \in \Gamma\right)$ есть элементарная теория алгебры $H$, т.е. множество таких формул $u \in \operatorname{Th}^{X}(H)$, что $\operatorname{Val}_{H}^{X}(u)=\operatorname{Hom}(W(X), H)$. Понятно также, что образ $\operatorname{Im} \operatorname{Val}_{H}$ является подалгеброй в $\mathrm{Hal}_{\Theta}(H)$, состоящей из одноопределенных множеств.

\section{7. Основная теорема.}

Tеорема 5.3 [5]. Многообразие $\mathrm{Hal}_{\Theta}$ порождено всеми алгебрами $\mathrm{Hal}_{\Theta}(H), H \in \Theta$.

Это означает, что тождества всех $\mathrm{Hal}_{\Theta}(H)$ задают многообразие алгебр Халмоша $\mathrm{Hal}_{\Theta}$.

Если $\Theta_{1}$ - подалгебра в $\Theta$, то многообразие $\mathrm{Hal}_{\Theta_{1}}$ в $\mathrm{Hal}_{\Theta}$, порожденное всеми $\mathrm{Hal}_{\Theta_{1}}(H)$, $H \in \Theta_{1}$, соответствует подалгебре $\Theta_{1}$. Следовательно, если $H_{1}$ и $H_{2}-$ алгебры из $\Theta_{1}$, то они изотипны в $\Theta_{1}$ тогда и только тогда, когда они изотипны в $\Theta$.

Сделаем также следующее общее наблюдение. Ясно, что найдется канонический гомоморфизм многосортных алгебр

$$
\pi_{H}: \Upsilon^{0} \rightarrow \operatorname{Hal}_{\Theta}(H),
$$

где $\pi_{H}=\left(\pi_{H}^{X}, X \in \Gamma\right)$ и $\pi_{H}^{X}-$ гомоморфизмы $\left.\pi_{H}^{X}: \Upsilon_{X}^{0} \rightarrow \operatorname{Bool}(W(X), H)\right)$. Этот гомоморфизм единственен, поскольку он переводит равенства в соответствующие равенства. Значит, ядро $\operatorname{Ker}\left(\pi_{H}\right)$ является также конгруэнцией тождеств алгебры $\operatorname{Hal}_{\Theta}(H)$. Рассмотрим $\widetilde{\pi}=$ $\bigcap_{H} \operatorname{Ker}\left(\pi_{H}\right)$. Это конгруэнция всех тождеств многообразия, порожденного всеми $\operatorname{Hal}_{\Theta}(H)$.

Теперь видно, что теорема 5.3 означает, что найдется равенство

$$
\operatorname{Hal}_{\Theta}^{0}=\Upsilon^{0} / \widetilde{\pi}, \quad \Phi(X)=\Upsilon_{X}^{0} / \widetilde{\pi}_{X},
$$

где $\widetilde{\pi}=\left(\widetilde{\pi}_{X}, X \in \Gamma^{0}\right)$ и $\widetilde{\pi}_{X}=\operatorname{Ker}\left(\pi_{X}\right)$. Конгруэнция $\widetilde{\pi}$ соответствует конгруэнции Линденбаума-Тарского.

В самом деле, пара формул $\left(u_{1}, u_{2}\right)$ принадлежит конгруэнции $\widetilde{\pi}_{X}$ тогда и только тогда, когда $u_{1}^{\pi_{H}^{X}}=u_{2}^{\pi_{H}^{X}}$ выполнено для любой алгебры $H \in \Theta$. Это означает также, что если $v-$ формула $\left(u_{1} \rightarrow u_{2}\right) \wedge\left(u_{2} \rightarrow u_{2}\right)$, то $v^{\pi_{H}^{X}}=1=\operatorname{Hom}(W(X), H)$. Это справедливо для всякого $X \in \Gamma^{0}$ и любой $H \in \Theta$, что дает искомое соответствие.

Мы привели необходимые сведения из алгебраической логики. Условия из п. 1.2 теперь реализованы. 
5.8. Категория элементарных множеств. Рассмотрим вначале категорию $\operatorname{Set}_{\Theta}(H)$ аффинных множеств над некоторой алгеброй $H$. Ее объекты имеют вид $(X, A)$, где $A-$ произвольное подмножество аффинного пространства $\operatorname{Hom}(W(X), H)$, а морфизмы имеют вид

$$
[s]:(X, A) \rightarrow(Y, B),
$$

где $s: W(Y) \rightarrow W(X)$ - морфизм в $\Theta^{0} ;$ соответствующее $\widetilde{s}: \operatorname{Hom}(W(X), H) \rightarrow \operatorname{Hom}(W(Y), H)$ должно быть согласовано с $A$ и $B$ следующим условием: если $\nu \in A \subset \operatorname{Hom}(W(X), H)$, то $\widetilde{s}(\nu) \in B \subset \operatorname{Hom}(W(Y), H)$. Тогда мы рассматриваем индуцированное отображение $[s]: A \rightarrow B$ как морфизм $(X, A) \rightarrow(Y, B)$.

Определим теперь категорию алгебраических множеств $K_{\Theta}(H)$ и категорию элементарных множеств $L K_{\Theta}(H)$. Обе эти категории - полные подкатегории в $\operatorname{Set}_{\Theta}(H)$ и понимаются как важные инварианты алгебры $H$. Назовем их AG- и LG-инвариантами алгебры $H$.

Объекты категории $K_{\Theta}(H)$ имеют вид $(X, A)$, где $A$ - алгебраическое множество в $\operatorname{Hom}(W(X), H)$. Если взять для $A$ элементарные множества, получится категория $L K_{\Theta}(H)$ элементарных множеств. Категория $K_{\Theta}(H)$ является полной подкатегорией в $L K_{\Theta}(H)$.

Как было упомянуто, если алгебры $H_{1}$ и $H_{2}$ изотипны, то категории $L K_{\Theta}\left(H_{1}\right)$ и $L K_{\Theta}\left(H_{2}\right)$ изоморфны.

5.9. Теория моделей и многосортная алгебраическая логика. Вспомним некоторые известные факты.

Начнем со следующего важного наблюдения. Наряду с алгеброй формул $\Phi=\Phi(X)$ рассмотрим алгебру чистых формул $\Upsilon_{X}^{0}$. Здесь формулы отождествляются с их записями. Сложность, возникающая в многосортном случае, состоит в следующем. В записи таких формул присутствуют не только переменные из множества (сорта) $X$. Это не позволяет использовать в категории $\Upsilon^{0}$ некоторые стандартные понятия теории моделей.

Следующие замечания показывают путь преодоления этого затруднения. Пусть $X-$ подмножество в $Y$, и пусть $s: X \rightarrow Y$ - тождественное отображение. Оно соответствует вложению $s_{*}: \Phi(X) \rightarrow \Phi(Y)$. Далее, пусть $u_{1} \in \Phi\left(X_{1}\right)$ и $u_{2} \in \Phi\left(X_{2}\right)$ - две формулы. Подмножества $X_{1}, X_{2}$ множества $Y$ вложены посредством $s^{1}: X_{1} \rightarrow Y$ и $s^{2}: X_{2} \rightarrow Y$. Формулы $u_{1} \in \Phi\left(X_{1}\right)$ и $u_{2} \in \Phi\left(X_{2}\right)$ назовем эквивалентнъмии, если для некоторого $Y \in \Gamma$ имеет место $s_{*}^{1}\left(u_{1}\right)=s_{*}^{2}\left(u_{2}\right)$. Формулу $u \in \Phi(X)$ назовем элементарной, если она принадлежит $\Phi^{0}(X)$. Доказано (см. [5]), что всякая формула в некотором $\Phi(X)$ эквивалентна некоторой элементарной формуле.

Для элементарных формул стандартные понятия теории моделей определяются естественным образом. В действительности, в теории моделей все формулы элементарны. Теорема из [5] позволяет рассматривать соответствующие стандартные понятия, используя эквивалентности формул.

В теории моделей переменная может быть либо связанной каким-то квантором, либо свободной. Это приводит к понятию замкнутой формулы. В многосортном случае также можно рассматривать замкнутые формулы.

Формула $u \in \Phi(X)$ называется замкнутой или предложением, если для всех $H \in \Theta$ значение $\operatorname{Val}_{X}^{H}(u)$ равно либо 0 , либо 1 в $\operatorname{Bool}(W(X), H)$ (т.е. либо всему пространству $\operatorname{Hom}(W(X), H)$, либо его пустому подмножеству). В односортном случае это определение совпадает с обычным.

Множество $T$ замкнутых формул в $\Upsilon^{0}$ или в $\mathrm{Hal}_{\Theta}^{0}$ называется теорией. Теория называется выполнимой, если она имеет модель. Теория $T$ называется полной, если для всякой замкнутой формулы $u$ либо $u$ принадлежит $T$, либо $\neg u$ принадлежит $T$. Теория $\operatorname{Th}(H)$ алгебры $H$ всегда полна. Теория $T$ называется категоричной в данной мощности $\alpha$, если любые две ее модели мощности $\alpha$ изоморфны.

Настоящая статья принадлежит к целому ряду статей об универсальной алгебраической геометрии (см. [4], [6]-[9], [15], [25] и др.). Как мы упомянули, в построениях рассматриваемой теории возникают различные вопросы, близкие к алгебре и теории моделей. Они кажутся 
новыми, хотя иные из них могут выглядеть простыми для специалистов в области теории моделей.

Следующие замечания касаются чистой логики и алгебраической логики в теории моделей. Теория моделей представляет собой комбинацию синтаксиса и семантики. Синтаксис (языки) играет в ней существенную роль, сравнимую с ролью семантики (моделей). Можно говорить о синтаксической структуре, относящейся к языкам, и о теориях в языках. Все это применимо к конкретным математическим задачам (см. [26]).

Алгебраическая логика это не влолне синтаксис. Она работает в теории моделей. Полезно использовать гомоморфизмы $\operatorname{Val}_{H}^{X}: \Phi(X) \rightarrow \operatorname{Bool}(W(X), H)$, соотносящие формулу и ее значение. Элементарную теорию можно представить как ядро такого гомоморфизма. Применительно к типам можно говорить о логическом ядре точки (т.е. гомоморфизма). Это ядро $\operatorname{LKer}(\mu)$ автоматически оказывается ультрафильтром в алгебре формул $\Phi=\Phi(X)$. Существует множество иных причин применять алгебраическую логику в теории моделей.

Еще раз перечислим открытые вопросы.

Проблема 5.4. Рассмотреть бесконечные логически нётеровы алгебры в различных многообразиях $\Theta$.

ПроблемА 5.5. Рассмотреть отделимые алгебры в различных многообразиях

ПроБлемА 5.6. Для каких многообразий $\Theta$ алгебры, свободные в $\Theta$, будут логически отделимыми?

ПроБЛЕма 5.7. Построить автоморфно финитарные алгебры $H$ в различных многообразиях $\Theta$. Другими словами, получить такие алгебры $H$ в $\Theta$, что для любого конечного $X$ в пространстве $\operatorname{Hom}(W(X), H)$ существует лишь конечное число $\operatorname{Aut}(H)$-орбит.

Проблема 5.8. Общая проблема: типы и изотипность в многосортных алгебрах. Рассмотреть эту проблему для многообразия представлений групп.

Отметим, что в [3] была поставлена проблема 1.23, касающаяся неизоморфных изотипных абелевых групп. Теперь мы видим, что эта проблема легко решается (предложение 3.4). На самом деле, для абелевых групп актуальна следующая проблема.

ПроБлЕма 5.9. Найти условия, при которых две абелевы группы элементарно эквивалентны, но не изотипны.

Наконец,

ПроБлемА 5.10. Какие нётеровы группы не являются логически нётеровыми?

Во всех этих случаях мы подразумеваем специфические $\Theta$ и $H \in \Theta$. Например, $\Theta$ могут состоять из модулей, векторных пространств, линейных алгебр, алгебр Ли, ассоциативных алгебр (конечной или бесконечной размерности) и т.д. Конкретное всегда иллюстрирует общее.

Автору приятно выразить благодарность Цлилю Села отметившему, что логическое ядро точки является типом, и Алексею Мясникову, обратившему внимание автора на тот факт, что логико-геометрическая эквивалентность алгебр есть не что иное, как изотипность алгебр. Автор признателен также своим коллегам Ю. Ершову, Е. Кацову, В. Ремесленникову, Э. Рипсу, А. Ольшанскому, Г. Житомирскому, Б. Зильберу и др. за постоянную поддержку.

Настоящая статья была написана в Юрмале, Латвия, где у автора были совершенные рабочие условия благодаря молодым друзьям Анне Ефименко и Диме Ковалю, а также Алле и Игорю Думанам. Рукопись была напечатана и подготовлена Е. и Т. Плоткиными. Автор выражает глубокую благодарность Д. И. Савельеву за перевод статьи на русский язык. 


\section{Список литературы}

[1] W. Hodges, Model Theory, Encyclopedia Math. Appl., 42, Cambridge Univ. Press, Cambridge, 1993.

[2] D. Marker, Model Theory. An Introduction, Grad. Texts in Math., 217, Springer-Verlag, New York, 2002.

[3] B. Plotkin, G. Zhitomirski, "Some logical invariants of algebras and logical relations between algebras", Алгебра и анализ, 19:5 (2007), 214-245; St. Petersburg Math. J., 19:5 (2008), 829-852.

[4] B. Plotkin, Seven Lectures on the Universal Algebraic Geometry, 2002, arXiv: math.GM/0204245.

[5] Б. Плоткин, “Алгебраическая геометрия в логике первого порядка", Алгебра и геометрия, Современная математика и ее приложения, 22, Институт кибернетики АН Грузии, Тбилиси, 2004, 16-62; J. Math. Sci. (N. Y.), 137:5 (2006), 5049-5097; arXiv: math.GM/0312485.

[6] B. Plotkin, "Some results and problems related to universal algebraic geometry", Internat. J. Algebra Comput., 17:5-6 (2007), 1133-1164.

[7] G. Baumslag, A. Myasnikov, V. Remeslennikov, "Algebraic geometry over groups. I. Algebraic sets and ideal theory", J. Algebra, 219:1 (1999), 16-79.

[8] A. Kvaschuk, A. Myasnikov, V. Remeslennikov, "Algebraic geometry over groups. III. Elements of model theory", J. Algebra, 288:1 (2005), 78-98.

[9] A. Myasnikov, V. Remeslennikov, "Algebraic geometry over groups. II. Logical foundations", J. Algebra, 234:1 (2000), 225-276.

[10] B. Plotkin, Algebraic Logic, Varieties of Algebras and Algebraic Varieties, arXiv: math.GM/0312420.

[11] P. R. Halmos, Algebraic Logic, Chelsea Publ., New York, 1962.

[12] Б. И. Плоткин, "Некоторые понятия алгебраической геометрии в универсальной алгебре", $A \Omega-$ гебра и анализ, 9:4 (1997), 224-248; St. Petersburg Math. J., 9:4 (1998), 859-879.

[13] R. Grossberg, "Classification theory for abstract elementary classes", Logic and Algebra, Contemp. Math., 302, Amer. Math. Soc., Providence, RI, 2002, 165-204.

[14] R. Baer, "Engelsche Elemente Noetherscher Gruppen", Math. Ann., 133:3 (1957), 256-270.

[15] Б. И. Плоткин, "Радикал и нильэлементы в группах", Изв. вузов. Матем., 1958, № 1, 130-135.

[16] В. I. Plotkin, "Notes on Engel groups and Engel elements in groups. Some generalizations", Изв. УрГУ, 2005, № 36, 153-166; arXiv: math.GR/0406100.

[17] Б. И. Плоткин, "Радикальные группы", Матем. сб., 37:3 (1955), 507-526.

[18] C.C. Chang, H. J. Keisler, Model Theory, Stud. Logic Found. Math., 73, North-Holland Publ., Amsterdam, 1973.

[19] G. Higman, B. H. Neumann, H. Neumann, "Embedding theorems for groups", J. London Math. Soc, 24:4 (1949), 247-254.

[20] А. Г. Курош, Теория груnn, Nauka, Moscow, 1967.

[21] P. Hall, "Some constructions for locally finite groups", J. London Math. Soc., 34:3 (1959), 305-319.

[22] O. H. Kegel, "Regular limits of infinite symmetric groups", Ischia Group Theory 2008, World Sci., Hackensack, NJ, 2009, 120-130.

[23] B. H. Neumann, "An essay on free products of groups with amalgamation", Philos. Trans. Roy. Soc. London. Ser. A, 246 (1954), 503-554.

[24] L. Henkin, J. D. Monk, A. Tarski, Cylindric Algebras. I, Stud. Logic Found. Math., 64, North-Holland Publ., Amsterdam, 1985; Cylindric Algebras. II, Stud. Logic Found. Math., 115, North-Holland Publ., Amsterdam, 1985.

[25] B. I. Plotkin, "Algebras with the Same (Algebraic) Geometry", Математическая логика и алгебра, Сборник статей. K 100-летию со дня рождения академика Петра Сергеевича Новикова, Тр. МИАН, 242, Наука, М., 2003, 176-207; Proc. Steklov Inst. Math., 242 (2003), 165-196; arXiv: math.GM/0210194.

[26] А. И. Мальцев, Алгебраические системь, Современная алгебра, Наука, М., 1970. 